\title{
Distribución y observaciones sobre la población de la nutria marina Lontra felina (Molina 1782) en el Perú
}

\author{
Distribution and observations on the population of marine otters Lontra \\ felina (Molina 1782) in Peru
}

\author{
Manuel Apaza ${ }^{1}$ y Leonardo Romero ${ }^{2}$
}

\begin{abstract}
1 Laboratorio de Ecología de Procesos, Facultad de CienciaBiología, Universidad Nacional Agraria La Molina, Av. La Molina s/n, La Molina, Perú

loxechinus@gmail.com

mal@lamolina.edu.pe

2 Departamento de Zoología, Facultad de Ciencias Biológicas, Universidad Nacional Mayor de San Marcos. Ciudad Universitaria, Av. Venezuela s/n. Apartado 110058, Lima 11, Lima Perú.

Iromeroc@unmsm.edu.pe
\end{abstract}

Presentado: $\quad 20 / 07 / 2011$ Aceptado: $\quad 23 / 01 / 2012$ Publicado online: $15 / 01 / 2013$

\begin{abstract}
Resumen
Analizamos la distribución de la nutria marina Lontra felina en la costa peruana, desde Punta Aguja $\left(05^{\circ} 47^{\prime} \mathrm{S}\right)$ hasta la frontera con Chile $\left(18^{\circ} 21^{\prime} S\right)$. Se realiza un análisis exhaustivo de la literatura referida a $L$. felina a fin de dilucidar un límite Norte de su distribución, concluyendo que no existe evidencia sólida sobre un posible límite Norte a los $6^{\circ} \mathrm{S}$. Por los mismos motivos se descarta la afirmación de una "distribución histórica" hasta la isla Lobos de Tierra. También es presentada información de un total de 272 nutrias registradas en 130 localidades entre los años 2000 al 2010. La distribución de estos registros no presenta autocorrelacion espacial sugiriendo una distribución homogénea. Tomando en consideración la morfoestructura y geomorfología de la costa peruana podemos observar coincidencias con la división en tres zonas: norte, centro y sur, las dos últimas presentan todos los registros de nutrias, y podrían ofrecer características del hábitat que permitirían una distribución continua de L. felina. Por último se señala un incremento en los valores del número de nutrias en la costa peruana al comparar los actuales con los reportados hace más de 40 años, aunque se observa la falta de trazabilidad de esta última información. Se sugiere desarrollar investigaciones que relacionen las abundancias y densidades de la nutria con la caracterización y distribución de sus hábitats, además de estudios de comportamiento que develen los procesos o caracteres intrínsecos de la especie para movilizarse en su área de distribución.
\end{abstract}

Palabras clave: especie amenazada; nutria marina; distribución geográfica; taxonomía.

\section{Abstract}

We analyzed the distribution of marine otter Lontra felina on the Peruvian coast, from Punta Aguja (05 $\left.47^{\prime} \mathrm{S}\right)$ to the boundary with Chile $\left(18^{\circ} 21^{\prime} S\right)$. We performed a comprehensive analysis of the literature on $L$. felina to elucidate a northern boundary of its geographic distribution, concluding that there is no solid evidence of a possible northern limit at $6^{\circ} \mathrm{S}$. For the same reasons, the affirmation of a "historic distribution" to the Lobos de Tierra island was discarded. It is also presented information of 272 otters from 130 locations between the years 2000 and 2010. The distribution of these records no has spatial autocorrelation, suggesting a homogeneous distribution. Considering the morphostructure and geomorphology of the Peruvian coast we can see similarities with the division into three zones: north, central and south, the last two add all records of otters, which could provide habitat characteristics that, allow a continuous distribution of $L$. felina. Finally there is an increase in the number of otters in the Peruvian coast to compare the current values with those of 40 years ago, but we show the lack of traceability of the latest information. We suggest undertaking research linking abundances and densities of otters with the characterization and distribution of their habitats, and behavioral studies that reveal the processes or intrinsic characteristics of the species to move into its geographic range.

Keywords: endangered species; marine otters; geographic distribution; taxonomy.

\section{Introducción}

Para evaluar el estado de amenaza de una especie y aplicar las medidas de conservación, es importante contar con información sobre la abundancia de las poblaciones (Nichols \& Williams 2006, Rodrigues et al. 2006, Taylor et al. 2007, Sinclair et al. 2009) y las variaciones en los rangos y límites de distribución geográfica (Brown et al. 1996, Sexton et al. 2009), al respecto, la nutria marina, Lontra felina (Molina, 1782), un carnívoro marino, distribuido desde el extremo sur de Argentina, todo el litoral de Chile hasta el Perú, presenta información discordante e imprecisiones sobre estos dos temas.

Desde finales del siglo XVIII la distribución de L. felina fue definida principalmente en base a observaciones aisladas, colectas de especímenes en expediciones y revisión de colecciones de museos. Aunque desde las últimas décadas del siglo XX a la fecha, otros aportes provenientes de observaciones e investigaciones más especificas han sido realizadas aún no han permitido consensuar el límite Norte de su distribución; que por registros de campo se ha establecido hasta los $9^{\circ} \mathrm{S}$ y en base a unas referencias bibliográficas de diverso origen algunos proponen a los $6^{\circ} \mathrm{S}$ o $6^{\circ} 27^{\prime} \mathrm{S}$.

Lontra felina es una especie listada en la Convención CITES (Apéndice I), clasificada como En Peligro por la UICN y legalmente protegida por el estado Peruano (Decreto Supremo No 034-2004-AG), condición que conllevó y motivó a realizar diversas investigaciones desde la pasada década, sin embargo las relacionadas con los aspectos poblacionales solo fueron en gran parte difundidas a través de informes inéditos y presentaciones en algunas reuniones especializadas.

Este trabajo, presenta los resultados de las evaluaciones de L. felina realizadas entre los ańos 2000 al 2010, y que en la literatura han sido usualmente referenciados por sus informes de origen y sobre los cuales se ha generado parte de la información sobre la densidad y tamaño de la población de $L$. felina en la costa peruana. En esta publicación, la primera de dos entregas, se analiza y discute esta información, contrastándola 
con la publicada y frecuentemente referenciada, precisando los valores poblacionales estimados de la nutria marina.

También, con nuestra información, observaciones y una revisión bibliográfica se sustenta porqué el límite Norte de distribución de L. felina se ubica en torno a los $9^{\circ} S$, también se discuten algunos aspectos particulares sobre su hábitat de desarrollo y como contribuyen también con esta posición.

\section{Material y métodos}

Distribución histórica de Lontra felina.- Realizamos una revisión de las publicaciones taxonómicas originales y las relacionadas con la distribución geográfica de L. felina desde su determinación inicial. La información pertinente fue resumida en una síntesis cronológica que incluyo al autor de la determinación original, la distribución y/o localidad tipo y los sinónimos asignados (Apéndice 1). Se especifica que una fuente no pudo ser consultada y solo se incluye el año, autor nombre científico, pero se incorpora en el resumen a fin de mantener la secuencia cronológica.

Registros de Lontra felina.- En el presente trabajo se presentan y analizan los datos originales obtenidos en las evaluaciones de la nutria marina en la costa peruana entre los años $2000 \mathrm{al}$ 2010. Los estudios se realizaron en la costa central y sur del Perú en el 2002, en la costa norte en el 2003, costa central y sur en el 2003, costa sur en el 2004, costa norte desde Punta Aguja hasta Nonura (Piura) en el 2007 y costa de Tacna y Moquegua en el 2008 y 2009 . Además se incluyen los registros de L. felina obtenidos en evaluaciones ambientales no específicas en la costa de Lambayeque (2001), costa sur de Piura (2002) y litoral de Lima (diferentes años). Los registros presentados fueron obtenidos por observación directa de individuos, no se consideraron datos indirectos como madrigueras, huellas, heces y animales muertos. Excepcionalmente fue incluida una comunicación personal.

Las posiciones geográficas de las localidades evaluadas fueron registradas con un GPS. Cuando no se dispuso de este dispositivo, la posición fue ubicada en las cartas geográficas del Instituto Geográfico Nacional (IGN). El sistema para referenciar las localidades fue el World Geodetic System 84 (WGS 84), expresado en coordenadas geodésicas cartesianas. Finalmente, se estableció como criterio base un periodo de 10 años de registros de individuos, porqué es el definido por las Listas Rojas de la UICN, para observar cambios de una población (UICN 2001). Esta convención se adoptó ante el desconocimiento de una real historia de vida de L. felina.

Análisis de la geomorfología de la costa del Perú.- Se elaboró un mapa base de perfil de la costa con las cartas nacionales del IGN (escala 1:100 000), en esta plataforma se delimitó un perfil de las unidades morfoestructúrales de la costa (INGEMMET 1995) y un tercero elaborado con la versión digital del Mapa Geomorfológico del Perú (http://geoservidor.minam.gob.pe/atlasperu/Default.asp ?WCI=PltEcosistemas\&WCE=1.1.2) a escala 1:200 000. El área de trabajo se estableció desde Punta Aguja $\left(05^{\circ} 47^{\prime} \mathrm{S}, 81^{\circ} 04^{\prime} \mathrm{W}\right)$ hasta la frontera con Chile $\left(18^{\circ} 21^{\prime} \mathrm{S}, 70^{\circ} 22^{\prime} \mathrm{W}\right)$. Punta Aguja fue elegida por ser el referente geográfico más conocido de Península Illescas, porque la posición de $06^{\circ} \mathrm{S}$, referenciada como límite norte de la distribución de L. felina, es un área sin referente geográfico conocido. Para el análisis se consideró un perímetro de $3080 \mathrm{~km}$ (INEI 2010), así como las posiciones de los límites de frontera, los especificados en la Ley No 28621 (2005).
Con los datos de las abundancias, mapa base de la costa y de las unidades geomorfológicas, se elaboró un mapa que presenta la distribución de las localidades de registros, número de individuos, además de las coberturas de las unidades morfo estructurales y de las unidades geomorfológicas de la costa peruana. Se elaboró una tabla con las longitudes del perímetro de la costa $(\mathrm{km})$ de cada unidad geomorfológica segmentada por cada grado de latitud desde Punta Aguja hasta la frontera con Chile. El mapa y la tabla fueron elaborados con el programa ArcGis v10.1.

Análisis estadísticos.- El análisis estadístico se realizó con los paquetes ape y vegan del programa $\mathbf{R}$ (http://cran.r-project. org/). Para la dispersión de los individuos de L. felina a lo largo de la costa peruana fue analizada con el Índice de Moran, algoritmo que pondera las distancias geográficas de los avistamiento y es ampliamente utilizado para detectar autocorrelación espacial (Sokal \& Oden 1978, Legendre \& Fortin 1989, Legendre \& Legendre 1998, Dormann et al. 2007). La distribución de las unidades geomorfológicas se analizó aplicando un agrupamiento jerárquico, a partir de una matriz de similaridad de índices de Bray-Curtis y el método de aglomeración complete linkage. Una vez observados los grupos, estos fueron considerados factores y la matriz de similaridad sometido a un Análisis de Similaridad (ANOSIM), este análisis es considerado un homólogo de un ANOVA de un factor (Clarke 1993)

\section{Resultados}

El límite de distribución histórico.- En la actualidad se referencian dos posiciones geográficas para establecer el límite Norte en la distribución de Lontra felina: la primera y más conocida se ubica en Chimbote, Ancash (ca. $9^{\circ} 04$ 'S), respaldada con registros de la especie y reportes más regulares en el tiempo. El segundo límite, recién propuesto para la Isla Lobos de Tierra, Piura (ca. $\left.6^{\circ} 27^{\prime} S\right)$ sustentada con una sola referencia (Schweigger 1964).

Desde los orígenes de la nomenclatura binomial publicado en el Systema Naturae (Lineo 1758) y con la determinación de la especie tipo Mustela lutra, se inició un periodo que hasta 1800 se caracterizó por diferenciar a las nutrias de los mustélidos dentro del género Lutra. Autores como Brisson (1762), Brünnichii (1772) y Erxleben (1777) son reconocidos en la determinación del género Lutra hasta que la ICZN (1998), dictaminó que la obra de Brisson se rechazaba para fines de nomenclatura por ser un trabajo no binomial, pero conservando el género en mención, y otros 11 para fines taxonómicos. También acorde con el nuevo esquema de clasificación, Molina (1782) nomino a la nutria marina como Mustela felina, especie incluida solo con su nombre común por Pennant (1793) sin aplicar el concepto binomial, pero nominada el mismo año por Shaw (1800) y Bechstein (1800) como Lutra felina con localidad tipo en Chili (Chile).

Desde el año 1800 hasta 1923, se describieron los sinónimos conocidos de L. felina, además de establecer la distribución de la especie según las localidades tipo referenciadas. Así Bennett (1832) describe a Lu chilensis, a partir de un espécimen colectado en la costa de Valparaíso (Chile). Posteriormente Gray (1837), determina a Lu. californica considerada como sinónimo de Lu. felina pero con una localidad tipo en California (USA?). En 1836, arribó al Callao (Perú) una expedición francesa, que colectó un espécimen de nutria en la isla San Lorenzo, que en 
1841 fue determinado por Paul Gervais como Lu. peruviensis, una nueva especie que relacionó con las nutrias del Pacífico Norte Lu. enhydris o Lu. lataxina, pero sin mencionar alguna relación con Lu. chilensis. También en 1841, Wagner determinó de manera temporal, un sinónimo de Lu. chilensis que nominó como Lu. brachydactyla, caso particular porqué fue una piel adquirida como "Lutra paranensis del oeste de América", que Wagner en una nota a pie de página consideró que su descripción estaba más relacionada con $L u$. chilensis, pero al observar diferencias en la morfología de los pies, la catalogó como Lu. brachydactyla, al final esta condición temporal terminó por ser definitiva.

En un primer ejercicio por organizar la taxonomía de las nutrias, Gray (1943) las dividió en función a dos categorías morfológicas: una agregaba a los géneros Enhydra y Pteronura, y la segunda a Latax, Aonyx, Lutra y Lontra, este último un nuevo género nunca aplicado hasta la propuesta de van Zyll de Jong (1972). Al ańo siguiente, en 1844, Tschudi publicó los resultados de sus exploraciones realizadas en el Perú entre 1838 y 1842, entre los mamíferos identificados, registró a Lu. chilensis y como sinónimo referenció a Lu. peruviensis (Gervais 1841). Sobre $L u$. chilensis, mencionó que fue encontrada en todos los lugares visitados de la Isla de Chiloé, costa de Chile y del Perú. Indicaba que los cazadores de focas (lobos marinos), la consideraban como rara y las sus pieles eran enviadas a Inglaterra desde Cobija, Iquique, Callao y Trujillo. Mencionó que Lu. chilensis también se encontraba en el Ecuador y probablemente en toda la costa occidental desde el archipiélago de Lemos $\left(45^{\circ} 12^{\prime} \mathrm{S}\right)$ hasta el sur de California (USA). Se observa que esta última información debió provenir de fuentes secundarias, porque Tschudi solo llegó hasta la caleta de Huacho $\left(11^{\circ} 07^{\prime} S\right)$ por la costa peruana (Tschudi 1846). Posteriormente, Gay en 1847 presentó una descripción más desarrollada de Lu. felina, además de identificar como sinónimos a Mustela felina de Molina y Lu. chilensis de Bennet.

Hasta 1865 las determinaciones de L. felina se distribuían desde Tierra de Fuego (Argentina), Chile y en el Perú hasta el Callao, excepto la referencia de Tschudi (1844). Pero Gray (1865) en otra revisión de la familia Mustelidae planteó la primera segregación de las especies del genero Lutra hacia Lontra (también descrito por Gray en 1843). Además de las especies asignadas al género Lontra, también incluye un nuevo género y especie: Nutria felina, pero le asigna una distribución desde Chile, isla de Chiloé, Perú, California (USA) y Kamchatka (Rusia). Gray sugirió también la presencia de la especie en Guatemala, por un espécimen colectado por Tomes (1861) que según Gray, concordaba con la descripción de Lu. chilensis de Waterhouse (1839), quién también determinó a Lu. platensis. Enfatizamos que los sinónimos referenciados por Gray (Lu. platensis y Lu. chilensis), fueron diferenciadas por el mismo Waterhouse en distribución y características morfológicas. Coues (1877) y Alston (1879 1882) repiten el error de reafirmar la extensa distribución de $L u$. felina establecida por Gray (1865) además incluyen también a Alaska, México, Guatemala, Costa Rica y Panamá.

Posteriormente Thomas (1889) publicó un trabajo enfocado a resolver inconsistencias en la taxonomía de la subfamilia Lutrinae y su sinonimia. Después de revisar diferentes fuentes y especímenes, estableció como área de distribución de Lu. felina el Estrecho de Magallanes, Patagonia, Chile y Perú, pero incurrió en un error al extender los limites hasta el Ecuador, porqué conocía un registro en "San Lorenzo". Esta era la localidad tipo de Lu. peruviensis que Gervais (1841) omitió en indicar como una isla en el catálogo de la especie, pero sí había sido nominada como una isla en otra sección del texto. Esta omisión orientó a Thomas a mencionar a "San Lorenzo", localidad costera de Ecuador, limítrofe con Colombia y dominada por manglares, sin embargo este error seria rectificado por Thomas 20 años después. Al documento de Thomas le sucedió el trabajo de Allen (1905), que asignó a Lu. felina sinónimos similares a los descritos por Thomas en 1889 , pero con una distribución que no incluyo al Perú. Con el propósito de precisar algunos alcances adicionales en la taxonomía de la subfamilia Lutrinae, en 1908, Thomas publicó otro trabajo sobre las nutrias de África y Sudamérica. Entre otros aspectos, presentar la descripción de 7 nuevas especies para Sudamérica en un esquema conocido como Grupo de Lu. platensis. En este esquema destacó Lu. provocax, como especie reconocida hasta la actualidad, y $L u$. incarium, especie con una localidad tipo en Cusco y un espécimen colectado en puerto Etén (costa norte del Perú), que Thomas relaciono con Lu. platensis como subespecie y actualmente un sinónimo de Lontra longicaudis.

Finalmente, se referencian dos especies de nutrias determinadas como sinónimos de L. felina en los años veinte. La primera, Lu. peruensis, fue una especie descrita por Pohle en 1920, que no ha sido revisada en este documento, pero se incluye en la cronología elaborada. La otra especie referenciada fue Lu. lutris, asignada al naturalista uruguayo Dámaso Antonio Larrañaga (1771 - 1848), pero recién publicada en un compendio conocido como Escritos, Tomo II en 1923. En realidad la especie referenciada por Larrañaga como la nutria del río La Plata fue Mustela lutris, que fue adaptada del sistema de clasificación de Cuvier. Sin embargo, Wozencraft (2005) referencia a Lu. lutris como sinónimo de L. felina del cuadro esquemático del sistema de Cuvier que presenta Larrańaga.

En este último periodo que se inició con el trabajo de Osgood (1943), se define con una distribución más precisa de L. felina desde la costa de Chile hasta el norte de Perú. También, en trabajos más recientes de Cabrera (1957), Harris (1968) y van Zyll de Jong (1972), determinan que L. felina se distribuye desde el Perú, Chile y extremo sur de Argentina, pero sin establecer una precisa ubicación del límite Norte. También Larivière (1998), reafirma este rango de distribución, pero comete un error al referir Chimbote a los $6^{\circ} \mathrm{S}$, cuando en realidad esta localidad se ubica a los $9^{\circ} \mathrm{S}$. Finalmente, Wozencraft (2005), mantiene la tendencia en la distribución, pero llama la atención sobre algunos sinónimos asignados, que anteriormente no habían sido referenciados.

Otras publicaciones no taxonómicas, también brindan información sobre el límite Norte de la distribución de L. felina. Así Koepcke (1958), señala su presencia hasta Perú central; Kostritsky (1963), que vive particularmente en el centro y sur de la costa peruana; Grimwood (1969) indica su registro hasta los $12^{\circ} \mathrm{S}$; Brack (1978) cita: "En el Perú está comprobada su existencia desde la costa central $\left(9^{\circ} \mathrm{S}\right)$, pero parece existir más al Norte, probablemente hasta los $6^{\circ} \mathrm{S}$ ”, en esa misma publicación, Brownell (1978) la ubica hasta Chimbote, y Goodwin \& Holloway (1978) en el IUCN Red Data Book, coincide con Pulido (1991) que la cita como: "probablemente su límite norte de distribución es Lima-Perú $\left(12^{\circ} \mathrm{S}\right)$ ”. Después, algunos 


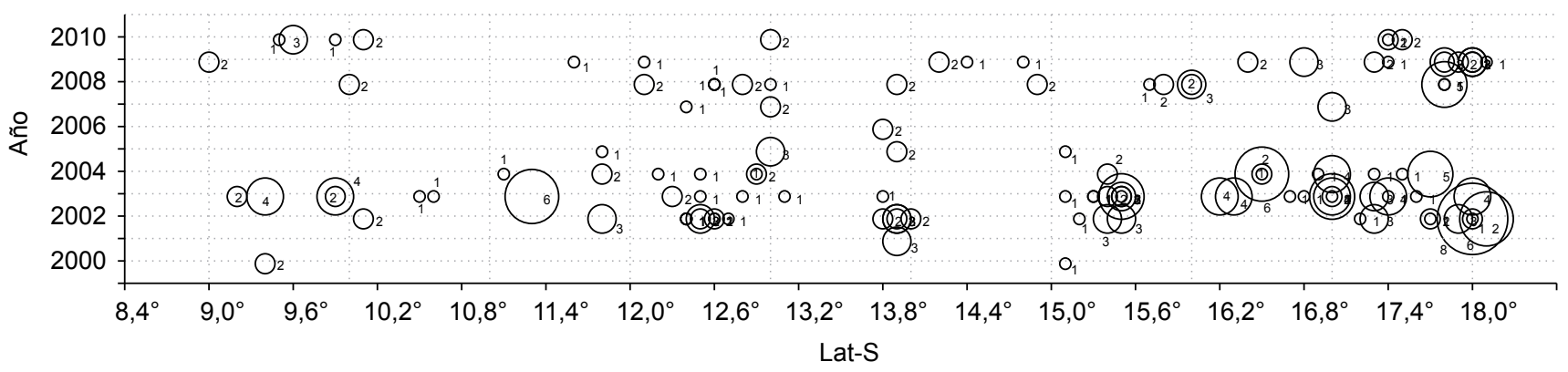

Figura 2. Gráfico de burbujas, mostrando el número de nutrias marinas, Lontra felina, avistas en cada registro (diámetro de los círculos), a lo

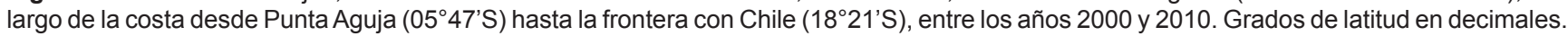

autores como Thornback \& Jenkins (1982), Cabello (1983) y Sielfeld (1990), comenzaron a citar la distribución hasta los $9^{\circ} \mathrm{S}$ y posiblemente hasta los $6^{\circ} \mathrm{S}$, otros la mantuvieron hasta los $9^{\circ} \mathrm{S}$ (Ebensperger y Castilla 1992, Reyes 1992), aunque Chehébar (1990) y Parera (1996) no dudaron en ubicarla hasta los $6^{\circ} \mathrm{S}$. Finalmente, en los últimos ańos algunas publicaciones referenciaron un límite Norte hasta los $6^{\circ} \mathrm{S}$ (Medina-Vogel, et al. 2004, Santibáńez 2005, Medina-Vogel et al. 2006, Medina-Vogel et al. 2007, Medina-Vogel et al. 2008, Badilla y George-Nascimento 2009, Córdova et al. 2009, Mangel et al. 2011).

Abundancia de Lontra felina.- Las observaciones sobre $L$. felina en el Perú generalmente han indicado que no son abundantes ni comunes (Coker 1908, Grimwood 1969, Brack 1978). También han sido consideradas como "poblaciones marginales", junto con las del centro y norte de Chile (Castilla \& Bahamonde 1979), en comparación con las mayores abundancias observadas en el sur de Chile, donde la literatura la referencia como común y abundante, hecho observado desde el viaje de Darwin en el HMS Beagle y otras expediciones (Waterhouse 1839, Allen 1905, Osgood 1943). En la actualidad la zona al sur de los $42^{\circ} \mathrm{S}$, sigue registrando las mayores densidades de L. felina (Cabello 1978, Sielfeld 1992, Sielfeld \& Castilla 1999, Medina-Vogel et al. 2006, Medina-Vogel et al. 2008).

En el Perú, desde finales de la década de los ochenta hasta el año 2000, se realizaron iniciativas para determinar la abundancia e identificar áreas de ocurrencia de L. felina, sin embargo, esta información en su mayoría no fue formalmente publicada y solo difundida a través de informes inéditos, comunicaciones personales y resúmenes (Sánchez 1990, Sánchez 1992, INRENA 1999, Riveros com. pers. en Majluf y Reyes 1989, CDC-UNALM 2000). Esta información fue referencia en publicaciones relacionadas a L. felina y temas marinos, pero solamente indicaban las áreas de registros sin incluir aspectos adicionales, como número de individuos registrados, características del hábitat o lo relacionado a metodologías o procedimientos de evaluación (Chehébar 1990, Reyes 1992, Vidal 1992).

Los resultados de las evaluaciones entre los años 2000 al 2010 registraron por observación directa, un total de 272 nutrias en 130 localidades de la costa peruana. Los registros se distribuyeron desde la desembocadura del río Santa $\left(08^{\circ} 58^{\prime} S\right)$ hasta las proximidades de la frontera con Chile. La ubicación de cada avistamiento se presenta en la Figura 1 y el detalle de cada registro, como departamento, localidad, coordenadas, año y número de individuos observados se enumera en el Apéndice 2. Se especifica que las evaluaciones en la costa de Lambayeque (2001), costa sur de Piura en Qda. Reventazón hasta Lambayeque en Pampa Las Salinas (2002) y costa norte desde Punta Aguja hasta Nonura (Piura) en el 2007, no registraron algún dato. Finalmente, un diagrama de burbuja grafíca una distribución homogénea de las abundancias a lo largo de este segmento de la costa peruana (Fig. 2). Para validar esta inferencia gráfica se aplicó el índice de Moran, calculado para el total de los avistamientos (2000 al 2010) y para periodos anuales con más de 15 avistamientos. En todos los casos el índice fue no significativo, lo cual prueba la falta de una autocorrelación espacial, e indica que no se forman agregaciones o conglomerados, y sugiere una distribución homogénea a lo largo del litoral peruano entre los $9^{\circ}$ a $18^{\circ} \mathrm{S}$ (Tabla 1). [Un análisis más detallado de esta distribución geográfica en relación a la estructuración espacial del hábitat se presenta en la segunda entrega de esta publicación].

Geomorfología de la costa peruana.- La distribución de las unidades geomorfologícas de la costa son presentados en la Figura 1. La longitud del perímetro de las unidades geomorfológicas desde Punta Aguja (0547'01.94”S, 8104'11.99”W) hasta la frontera con Chile (18 $21^{\circ} 08.00^{\prime \prime}$, $\left.70^{\circ} 22^{\prime} 39.00^{\prime \prime W}\right)$ cubren un total de $2560,9 \mathrm{~km}$. Este perímetro desagregado por intervalo de grado de latitud para las siete unidades geomeorfológicas consideradas muestra claras diferencias en la distribución a lo largo del área estudiada (Tabla 2). Al aplicar un análisis de agrupamiento jerárquico obtenemos un dendrograma donde se aprecian cinco grupos que presentan diferencias significativas (ANOSIM, R= 0,91; $<<0,001 ;$ Permutaciones= 99999) (Fig. 3).

En la Figura 1, también se aprecia el perfil de las unidades morfoestructúrales de la costa peruana que grosso modo segmenta la costa desde frontera con Chile hasta Punta Pejerrey (Cordillera de la Costa) y desde Punta Pejerrey hasta casi la Península Illescas (Planicie Costera) donde un fragmento emergente de la Cordillera Occidental completa este segmento. El

Tabla 1. Valores calculados del Indice de Moran. Observado: I Moran calculado, Esperado: el valor esperado de I Moran bajo la hipótesis nula, Sd: la desviación estándar de I Moran p.value: el P-valor de la prueba de la hipótesis nula contra la hipótesis alternativa especificada en alternativa.

\begin{tabular}{cccccc}
\hline Periodo & $\mathrm{n}$ & observado & experado & $\mathrm{sd}$ & p.value \\
\hline $2000-10$ & 130 & 0,0689 & $-0,0078$ & 0,0444 & 0,0844 \\
2002 & 28 & 0,0183 & $-0,0370$ & 0,1073 & 0,6062 \\
2003 & 34 & $-0,0703$ & $-0,0303$ & 0,0992 & 0,6871 \\
2004 & 15 & $-0,1989$ & $-0,0714$ & 0,1564 & 0,4150 \\
2008 & 16 & $-0,0045$ & $-0,0667$ & 0,1531 & 0,6850 \\
2009 & 18 & 0,1156 & $-0,0588$ & 0,1543 & 0,2582 \\
\hline
\end{tabular}




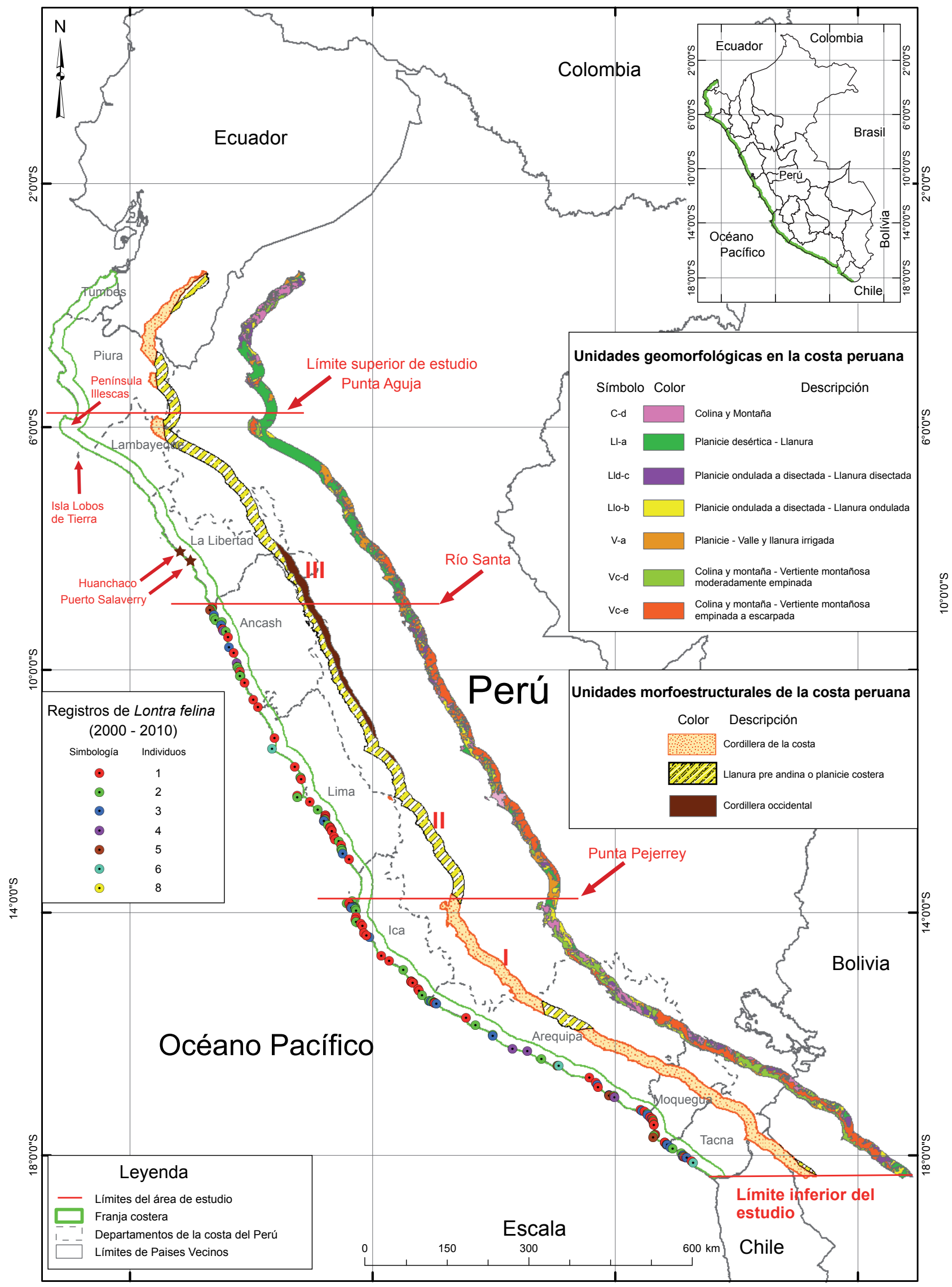

Figura 1. Perfiles costeros de las unidades morfoestructurales y unidades geomorfológicas. Se indican la ubicación y número de avistamientos de Lontra felina. Se señala la ubicación de la isla Lobos de Tierra, los reportes de nutria en los $8^{\circ} 11$ 'S (Puerto Salaverry - La Libertad, Santillán \& Caro, 2007) y a los $8^{\circ} 04^{\prime} S$ (Huanchaco - La Libertad, Alfaro et al. 2011). 
Tabla 2. Longitudes del perímetro $(\mathrm{km})$ de cada unidad geomorfológica segmentada por cada grado de latitud desde Punta Aguja hasta la frontera con Chile. C-d: Colina y montaña, LI-a: Planicie desértica - Llanura, Lld-c: Planicie ondulada a disectada - Ilanura, disectada, Llob: Planicie ondulada a Disectada - Llanura ondulada, V-a: Planicie - Valle y Llanura irrigada, Vc-d: Colina y Montaña - Vertiente Montañosa moderadamente empinada, Vc-e: Colina y Montaña - Vertiente Montañosa empinada a escarpada.

\begin{tabular}{|c|c|c|c|c|c|c|c|c|}
\hline \multirow{2}{*}{$\begin{array}{c}\text { Latitud Sur } \\
\text { (grados decimales) }\end{array}$} & \multicolumn{7}{|c|}{ Unidades Geomorfológicas } & \multirow{2}{*}{$\begin{array}{c}\text { Perímetro } \\
\text { km }\end{array}$} \\
\hline & C-d & Ll-a & Lld-c & Llo-b & V-a & Vc-d & Vc-e & \\
\hline $05,78^{\circ}-07,00^{\circ}$ & 5,2 & 173,2 & 0,0 & 6,6 & 2,1 & 36,3 & 0,0 & 223,4 \\
\hline $07,00^{\circ}-08,00^{\circ}$ & 5,6 & 93,5 & 0,0 & 24,4 & 19,8 & 0,0 & 0,0 & 143,3 \\
\hline $08,00^{\circ}-08,98^{\circ}$ & 0,0 & 84,6 & 0,0 & 15,5 & 33,2 & 0,0 & 9,5 & 142,8 \\
\hline $08,98^{\circ}-10,00^{\circ}$ & 8,5 & 24,2 & 39,2 & 43,7 & 11,5 & 0,0 & 125,6 & 252,7 \\
\hline $10,00^{\circ}-11,00^{\circ}$ & 38,5 & 23,5 & 4,0 & 33,4 & 36,5 & 0,0 & 23,0 & 158,8 \\
\hline $11,00^{\circ}-12,00^{\circ}$ & 46,5 & 37,9 & 0,0 & 14,2 & 44,7 & 18,6 & 7,6 & 169,6 \\
\hline $12,00^{\circ}-13,00^{\circ}$ & 52,8 & 36,5 & 1,5 & 22,0 & 45,2 & 20,4 & 0,0 & 178,3 \\
\hline $13,00^{\circ}-13,47^{\circ}$ & 9,8 & 29,1 & 0,0 & 10,9 & 70,6 & 7,4 & 0,0 & 127,8 \\
\hline $13,47^{\circ}-15,00^{\circ}$ & 46,4 & 0,0 & 0,0 & 74,6 & 0,0 & 110,3 & 31,4 & 262,6 \\
\hline $15,00^{\circ}-16,00^{\circ}$ & 54,4 & 0,0 & 44,2 & 46,4 & 2,5 & 95,6 & 23,4 & 266,5 \\
\hline $16,00^{\circ}-17,00^{\circ}$ & 0,0 & 0,0 & 0,0 & 31,0 & 29,2 & 216,4 & 45,2 & 321,9 \\
\hline $17,00^{\circ}-18,00^{\circ}$ & 0,0 & 5,5 & 0,0 & 5,9 & 47,2 & 172,6 & 7,2 & 238,4 \\
\hline $18,00^{\circ}-18,35^{\circ}$ & 0,0 & 32,5 & 0,0 & 9,7 & 6,4 & 2,9 & 23,6 & 75,0 \\
\hline Perímetro (km) & 267,5 & 540,4 & 88,9 & 338,2 & 348,8 & 680,6 & 296,5 & 2560,9 \\
\hline
\end{tabular}

agrupamiento de las unidades geomorfológicas observadas en el dendrograma presenta una correspondencia con las unidades de la morfoestructura (Fig. 3). El grupo morfoestructural I (Ay B geomorfológico) agregó el 69,5\% de los registros de L. felina, mientras el grupo II (C y D geomorfológico) el 30,5\%, y el grupo III (E geomorfológico), que estructuralmente parecía ser similar al anterior pero no presento registros.

Estimados de población de Lontra felina en el Perú.- Una información recurrente sobre la población de nutrias marinas en el Perú estima para su distribución en la costa peruana entre 200 a 300 individuos. Las referencias más conocidas o las consideradas de origen son: Grimwood (1967), Harris (1968), Grimwood (1969), IUCN 1970 (en Brownell 1978), ACMRR/ FAO (1976), IUCN (1976, en Goodwin \& Holloway 1978), Vaz-Ferreira (1979) y Castilla y Bahamondes (1979), este último

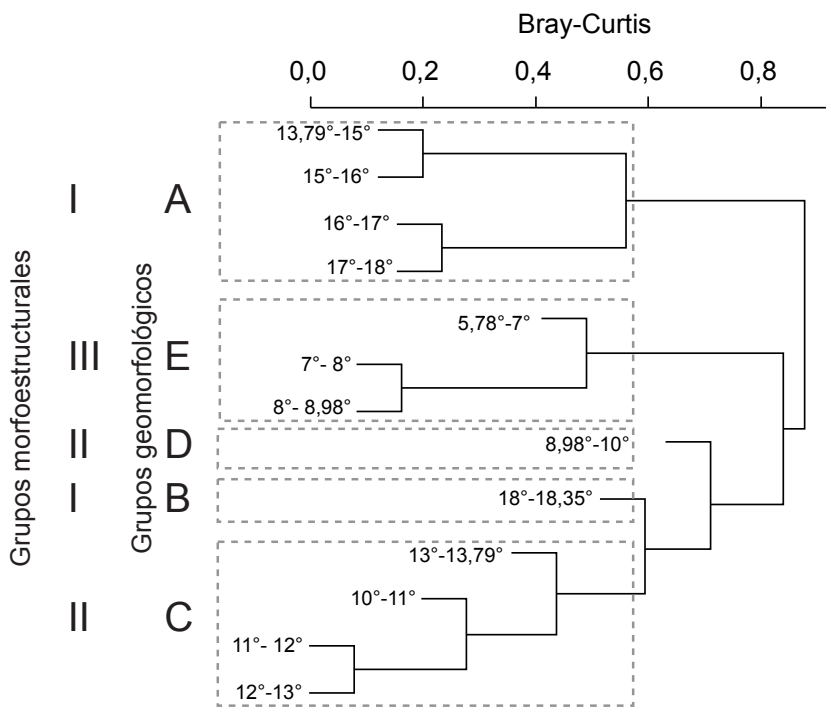

Figura 3. Dendrograma de grupos geomorfológicos. Analisis de agrupamiento jerárquico a partir de una matriz de similaridad de índices de Bray-Curtis y el método de aglomeración complete linkage. Se utilizan los perímetros de las unidades geomorfológicas agrupados por cada intervalo de grado de latitud. Se indican los grupos geomorfológicos formados (A, B, C, D, E) y también los morfoestructurales ((I, II, III). autor por ejemplo referencia el dato del IUCN Red Data Book (1976), que a su vez se atribuye el dato a Harris (1968) y una comunicación personal de Grimwood (1967), como respaldo de la información. Sin embargo, ninguna de las publicaciones citadas es la fuente de origen de este cálculo poblacional. Insistiendo en el tema, se revisaron todas las publicaciones de Grimwood (1967, 1968a, 1968b y 1969) y no fue posible ubicar el dato poblacional mencionado. Sobre esta revisión deducimos que el estimado de población sería una comunicación personal o una errada transcripción de alguno de los documentos señalados. Algo semejante ocurre con el estimado de 1000 individuos (FAO-ACMRR. 1978, Vaz-Ferreira 1979) para toda el área de distribución de L. felina, que Sielfeld y Castilla (1999) observan, porque en similar condición, no conocen su cálculo y además la consideran como una cantidad que subestima la población de L. felina en la región.

A partir del año 2000 se inician algunas evaluaciones orientadas a determinar la abundancia e identificar las áreas de ocurrencia de L. felina. Así, Apaza et al. (2002) al determinar una densidad promedio de 2,0 ind $/ \mathrm{km}$, y considerando un perímetro de $520 \mathrm{~km}$ como ambiente potencial para la nutria marina, entre la zona entre Lima y Tacna y textualmente citó: "un grosero estimado del número de individuos en el litoral sur del Perú que alcanzaría a los 620 individuos”. En el informe se omitió especificar que del perímetro de $520 \mathrm{~km}$ solo se consideraron $310 \mathrm{~km}$, por eso solo se mencionó solo el litoral Sur y la posición geográfica de referencia se ubicó aproximadamente en el extremo norte de la Península de Paracas (1347'S), en la Reserva Nacional de Paracas, Dpto. de Ica.

Posteriormente, después de realizar sucesivas evaluaciones hasta el 2004, la densidad determinada en el 2002, se ajustó a 2,21 ind/ km (CPPS 2004) y se estimó un número potencial de nutrias en función al hábitat disponible $(312 \mathrm{~km})$ que alcanzó los 690 76 individuos (Apaza et al. 2004) desde Ica (1347’S) hasta Tacna $\left(18^{\circ} 21^{\prime} \mathrm{S}\right)$. Se observa otra vez, que el estimado estuvo dirigido por un cálculo espacial y no por un conteo de especímenes, razón por lo que fue denominado como potencial y no un estimado de población como es mencionado en diversas publicaciones. 
A diferencia de la omisión del informe del 2002, sobre el rango de cobertura, en el 2004, en el texto se incurrió en un error al especificar el área entre Lima y Tacna, sin embargo las cantidades o medidas afortunadamente no fueron afectadas por el desacierto.

Mientras que en 2004, el número de nutrias para Perú fue calculado utilizando la densidad promedio de siete localidades (que solo representan $32,5 \mathrm{~km}$ de costa evaluados), distribuidas entre los departamentos de Ica a Tacna, considerando $312 \mathrm{~km}$ de litoral como hábitat potencial para L. felina y el registro de 32 individuos; en el 2005 en un ejercicio más elaborado, se incluyeron 78 localidades, que correspondían a 114 kilómetros de litoral, entre los departamentos de Lima $\left(11^{\circ} 46\right.$ 'S) y Tacna $\left(18^{\circ} 21^{\prime} \mathrm{S}\right)$, considerando $510 \mathrm{~km}$ de litoral como hábitat potencial para nutrias y 169 individuos registrados, además de un trabajo cartográfico más preciso. Los resultados mostraron obvias diferencias, dando como resultado una densidad promedio de $1,48 \mathrm{ind} / \mathrm{km}$ y una abundancia total de $756 \pm 86$ individuos (Apaza et al. 2005).

Recientemente, Valqui (2012) ensayó un estimado adicional de población y propuso que estaría fluctuando entre 789 y 2131 individuos para toda la costa del Perú, calculados sobre $789 \mathrm{~km}$ de costa que sería el probable hábitat de L. felina y con densidades entre 1,0 - 2,7 ind $/ \mathrm{km}$. El autor argumentó que los valores usados fueron un consenso de las diferentes densidades publicadas para Perú y Chile, como referencia de la información menciona a sus observaciones entre los años 2008 y 2011, además de los datos otros autores. Se observa que el autor no especifica en la publicación, el rango geográfico de cobertura, además si el segmento potencial $(789 \mathrm{~km})$, es una línea recta trazada desde los extremos del rango de cobertura o el perímetro litoral de esa sección.

\section{Discusión}

La revisión de la literatura desde el origen de la determinación de L. felina hasta el presente no muestra evidencia sólida que permita afirmar que existió una "distribución histórica" de nutria marina hasta la isla Lobos de Tierra $\left(6^{\circ} 27^{\prime} S\right)$ o en torno a Península Illesca (ca. 6오's).

Se han observado sucesivas confusiones en diferentes momentos de la historia, como la errada interpretación del trabajo de Tschudi (1844), de una distribución hasta el Ecuador; o el trabajo de Thomas (1889) al confundir la localidad de la isla San Lorenzo con una homónima en la costa de Ecuador [aunque Thomas (1908) rectifica la ubicación en el Callao-Perú], esta confusión probablemente afecto el trabajo de Quijada (1910) que reseña la distribución de la nutria marina hasta Ecuador. Si bien el tiempo podría haber aclarado esta confusión, como la que ocurrió en el siglo XVIII cuando se consideraba una distribución hasta Rusia y toda la costa Occidental de América (Gray 1865, Coues 1877, Alston 1879-1872), otra vez en el siglo XX, una distribución referida como probable hasta los $6^{\circ} \mathrm{S}$ (Brack 1978), sumado al trabajo de Larivière (1998) que erradamente localiza a Chimbote $\left(9^{\circ} \mathrm{S}\right)$ a los $6^{\circ} \mathrm{S}$, parecen haber tenido, un efecto sinérgico por la amplia difusión de ambos trabajos y que ha terminado por causar hechos anecdóticos como el que se observa actualmente en dos portales de la UICN para L. felina: que en la Lista Roja de Especies Amenazadas se referencia el límite norte hasta los $6^{\circ} \mathrm{S}$, y en el Otter Specialist Group (OSG) en la localidad de Chimbote a los $9^{\circ} \mathrm{S}$.
Recientemente, Valqui (2012) citó a Schweigger (1964), quién menciona a L. felina en el "grupo de las islas Lobos", pero sin embargo, en la primera edición de su libro (Schweigger 1947), identificó al "gato marino" como posiblemente Latax sp. un sinónimo del genero Enhydra al que pertenece la nutria de California E. lutris. También resaltamos que la designación "islas Lobos" es utilizado en otros grupos insulares menores de la costa. Además el trabajo de Schweigger no fue el producto de un estudio dirigido a evaluar a la nutria o la fauna del cuerpo insular, como por ejemplo la evaluación de la fauna, que entre otras regiones de la costa Coker (1908), realizó en la Isla Lobos de Tierra y donde no menciona la presencia de L. felina.

Por otro lado, Alfaro-Shigueto et al. (2011), argumentan que la falta de constancia en los registros históricos durante el siglo XX ha generado este diferendo en la distribución de L. felina, y también basa la distribución de la especie hasta la Isla Lobos de Tierra $\left(6^{\circ} 25^{\prime} S\right)$ por el documento de Schweigger (1964), y sostiene que diversos impactos antropogénicos han originado la contracción en la distribución en aproximadamente $400 \mathrm{~km}$, desde la Isla Lobos de Tierra hasta Chimbote $\left(9^{\circ} 10^{\prime} \mathrm{S}\right)$.

$\mathrm{Al}$ integrar todos los elementos expuestos, podemos deducir que la opinada contracción o perdida de la distribución de $L$. felina al norte del río Santa, esta sustentada más por errores en la historia de las nominaciones taxonómicas, comunicaciones imprecisas entre otros, pero que de acuerdo a lo investigado en este trabajo, no representan argumentos sólidos que respalden un alcance hasta los $6^{\circ} \mathrm{S}$ o una reciente "distribución histórica" de L. felina hasta la isla Lobos de Tierra $\left(6^{\circ} 25^{\prime}\right)$.

La información de los 272 registros originales de L. felina en 130 localidades a lo largo de la costa presentados en este trabajo, muestran una distribución homogénea, es decir que las fluctuaciones del número de individuos y su presencia en el espacio ocurren al azar, no existiendo indicios de lugares con mayor o menor concentración de individuos. Sin embargo, anotamos que esta información no incluyen los registros indirectos, ni los registros de otros investigadores lo cual podría reforzar la afirmación de una distribución homogénea de L. felina en la costa peruana.

El análisis de la distribución de las unidades geomorfológicas (Fig. 3) mostró tres agregaciones bien diferenciadas: (A) desde

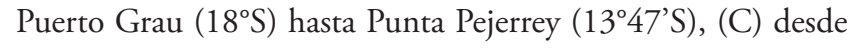
Punta Pejerrey (134ㄱ' hasta próximo a Huarmey $\left(10^{\circ} \mathrm{S}\right)$, y desde el río Santa $\left(08^{\circ} 58^{\prime} S\right)$ hasta Punta Aguja (05\%47’S), además de los subgrupos: (D) del río Santa $8^{\circ} 58^{\prime} S$ hasta Huarmey $\left(10^{\circ} \mathrm{S}\right)$ y $(\mathrm{B})$ desde Puerto Grau $\left(18^{\circ} \mathrm{S}\right)$ hasta frontera con Chile $\left(18^{\circ} 21^{\prime} S\right)$. Si bien se observan diferencias las zonas del centro y sur de la costa peruana (Fig dendrograma, A y C) esto no afectaría la distribución de las nutrias. Un elemento importante del hábitat para L. felina serían sus madrigueras (Sielfeld \& Castilla 1999), que se ubican entre las rocas de derrumbe de acantilados rocosos y playas pequeńas, en cuevas con galerías o túneles que tienen salida al mar, usan las rocas expuestas para descansar, acicalarse, solearse y recrearse. Este tipo de hábitat podría distribuirse homogéneamente desde el sur hasta el río Santa $\left(08^{\circ} 58^{\prime}\right.$ 'S), o por lo menos encontrarse con facilidad. Sin embargo hacia el norte predomina la Planicie Costera, en la cual estos hábitats no existirían o serian escasos. Los reportes de $L$. felina para Huanchaco ( $8^{\circ} 04^{\prime} S$ ) en La Libertad (Alfaro et al. 2011) y de Puerto Salaverry ( $8^{\circ} 13^{\prime} S$ ) en La Libertad (Santillán 
y Caro, 2007) coinciden con una zona de transición antes de la Planicie Costera. Estos dos reportes son utilizados como un indicio de la "reciente distribución histórica", pero la información geomorfológica indicaría que serían algo esperado el encontrar nutrias en esa zona, sin inferir sobre posibles valores o estimados de abundancia en esa región, pero si donde procesos naturales estarían actuando como limitante de la distribución por contener no solo una menor área disponible de hábitat terrestre para $L$. felina sino además limitada tolerancia a variables o fluctuaciones climato-oceanográficas de esa región, antes que atribuirse a factores antrópicos recientes.

La afirmación de que la población de L. felina esta decreciendo y que puede leerse en la ficha de la IUCN (Alvarez \& Medina-Vogel 2008) discrepa del aparente crecimiento según los datos estimados publicados (200 - 300 ind. en la década de los 60 - 70?; 620 ind. 2002, costa sur del Perú; 690 ind. 2004, costa sur del Perú; 756 ind. 2005, costa central y sur del Perú y 789 ind. 2012, costa peruana). Estos estimados en su mayoría carecen de trazabilidad, y no podrían ser utilizados en modelos de población, pero en general los publicados en los últimos 10 años, representan un estado real de su población respecto a los supuestos hace más de 40 años.

Nuestro análisis y revisión propone que la estimación del número de individuos de L. felina en la costa peruana debe responder a propuestas que relacionen las abundancias y densidades de la nutria con la caracterización y distribución de sus hábitats, además de estudios de comportamiento que develen los procesos o caracteres intrínsecos de la especie para movilizarse dentro su área de distribución. Esta última inferencia se asume de los estudios genéticos que destacan una alta variabilidad en los análisis realizados en el ADN de L. felina, probablemente alcanzado por el intercambio regular de individuos aun en regiones que aparenten ser cuellos de botella en la distribución (Valqui 2010, Vianna 2010).

Esta primera entrega ha pretendido actualizar la información referente a los aspectos de distribución y población de L. felina, con algunos alcances del medio terrestre donde se desarrolla. Que en la segunda entrega se enfocara en caracterizar el hábitat espacial de L. felina, así como los factores que determinan la estructura de la población a lo largo de la distribución, en una escala espacial de análisis más específica a la presentada.

\section{Agradecimientos}

A la Blga. Rosario Acero V. por el valioso apoyo brindado durante su gestión en el ex-INRENA (ahora Dirección General Forestal y de Fauna Silvestre-MINAG), también a Matthew Rutishauser, Friends of the Sea Otter (Science Director 2002). Por brindar información bibliográfica a Katherine Rewinkel, Librarian, Global Communications Unit-IUCN, Ricardo Muñoz, David Lubin Memorial Library-FAO, Verónica Ávila, Oficina Coordinación Regional del Plan de Acción-CPPS, Blga. Daniela Laines, Blga. Carolina Tovar, Dr. Víctor Pacheco (MHN-UNMSM), Bs. Cindy Hurtado e Ing. Antonio Tovar N. (información y dato del Regatas). Al Dr. Reynaldo LinaresPalomino por su apoyo con los textos en alemán

\section{Literatura citada}

ACMRR / FAO. 1976. Mammals in the Seas: Ad Hoc Group III on Seals and Marine Otters (draft report). Advisory Committee on Marine Resources Research and Food and
Agriculture Organization of the United Nations. Scientific Consultation on Marine Mammals (FAO-FI-ACMRR/ $\mathrm{MM} / \mathrm{SC} / 4)$. Bergen, Norway. 182 p. + Supplement \& Addendum.

Alfaro-Shigueto J., J. Valqui \& J.C. Mantel. 2011. Nuevo Registro de la Nutria Marina Lontra felina (Molina, 1782) al Norte de su Distribución Actual. Ecol. Apl. 10(2):87-91.

Allen J.A. 1905. Mammalia of Southern Patagonia. In W. B. Scott (ed.). Report of the Princeton University Expeditions to Patagonia, 1896 - 1899. Zoology. Princeton, N.J. The University. Vol. 3. 210 p. 29 pl.

Alston E.R. 1879-1882. Mammalia. Biologia Centrali-Americana. Taylor and Francis. London, United Kingdom. 220 p. 22 pl.

Alvarez R. \& G. Medina-Vogel. 2008. Lontra felina. In: IUCN 2012. IUCN Red List of Threatened Species. Version 2012.2. $<$ www.iucnredlist.org>. <acceso 10/12/2012).

Alvarez R., C. Delgado \& J. Ruíz. 2009. Distribution and Conservation Status of Lontra felina (Molina, 1782) Along of Chiloense Marine Ecoregion, Chile. pp: 14-15. Abstract Book. In: 10th International Mammalogical Congress, Mendoza, Argentina, 9-14 August 2009. 356 p.

Apaza, M., M. Lleellish, J. Valqui, C. Céspedes, M. Roca, J. Alfaro y G. Munemura. 2002. Estado de Conservación de las Poblaciones de Spheniscus humboldti y Lontra felina en la Costa Peruana. Instituto Nacional de Recursos Naturales (INRENA). Informe Final. Lima. 37 p. (Informe Inedito).

Apaza, M., J. Valqui, J. Mangel, M. Roca, et al. 2004. Lontra felina (Molina 1872), Situación Actual en el Perú. Libro de Resúmenes pp: $4.11^{\mathrm{a}}$ Reunión de Trabajo de Especialistas en Mamíferos Acuáticos de América del Sur y $5^{\circ}$ Congreso de la Sociedad Latinoamericana de Especialistas en Mamíferos Acuáticos (SOLAMAC). Setiembre del 2004, Quito - Ecuador. (Resumen).

Apaza, M., Valqui, J. Mangel, J. Perret, et al. 2005. Population Density and Threats Analysis of Marine Otters Lontra felina (Molina 1782) in the Central and Southern Coast of Peru. pp: 16 - 17. Abstract Book. In: 16th Biennial Conference on the Biology of Marine Mammals. Society for Marine Mammalogy. San Diego, California, 12 - 16 December. USA. 330 p. (Abstract)

Badilla, M y M. George-Nascimento. 2009. Conducta diurna del chungungo Lontra felina (Molina, 1782) en dos localidades de la costa de Talcahuano, Chile: ¿efectos de la exposición al oleaje y de las actividades humanas? Revista de Biología Marina y Oceanografía 44: 409-415.

Baird S.F. 1855. List of Mammalia found in Chili. Vol 2: 163-171. In J. M. Gillis (Ed.). The U.S. Naval Astronomical Expedition to the Southern Hemisphere, During the Years 1849-'50-'51-'52. 23d. Congress, 1st. Session. House of Representatives. Ex. Doc. N ${ }^{\circ}$ 121. A. O. P. Nicholson, Printer. Washington. Vol. 2. 300 p.

Bechstein J.M. 1800. Thomas Pennant's allgemeine Übersicht der Vierfüssigen Thiere. Aus dem englischen übersetzt aus mit Anmerkungen und Zusätzen versehen von J. M. Bechstein. Vol. 2. Landes-Industrie-Comptoir, Weimar. 666 p.

Bennett E.T. 1832. Characters of a New Species of Otter (Lutra, ErxL), and of a New Species of Mouse (Mus, L.), collected in Chili by Mr. Cuming. Proceedings of the Committee of Science and Correspondence of the Zoological Society of London. Printed by Richard Taylor. Part 2. pp: 1-2

Brack A. 1978. Situación Actual de las Nutrias (Lutrinae, Mustelidae) en el Peru. pp: 76-84. In N. Duplaix (Ed.). Otters: Proceedings of the Firts Working Meeting of the Otter Specialist Group. WWF / SSC / IUCN. Paramaribo, Suriname 27-29 March 1977. Internacional Union for Conservation of Nature and Natural Resources, Publication New Series. Morges, Switzerland. $158 \mathrm{p}$.

Brisson M.J. 1762. Regnum animale in classes IX. distributum, sive synopsis methodica sistens generalem animalium 
distributionem in classes IX, \& duarum primarum classium, quadrupedum scilicet \& cetaceorum, particularem divisionem in ordines, sectiones, genera \& species. Cum brevi cujusque speciei descriptione, citationibus auctorum de iis tractantium, nominibus eis ab ipsis \& nationibus impositis, nominibusque vulgaribus. Lugduni Batavorum. Apud Theodorum Haak. Editio altera auctior. 296 p.

Brown, JH, GC Stevens, y DW Kaufman. 1996. The geographic range: size, shape, boundaries and internal structure. Annual Review of Ecology and Systematics 27: 597-623.

Brownell. R.L. 1978. Ecology and Conservation of the Marine Otter, Lutra felina. pp: 104-106. In N. Duplaix (Ed.). Otters: Proceedings of the Firts Working Meeting of the Otter Specialist Group. WWF / SSC / IUCN. Paramaribo, Suriname 27-29 March 1977. Internacional Union for Conservation of Nature and Natural Resources, Publication New Series. Morges, Switzerland. $158 \mathrm{p}$.

Brünnichii M.Th. 1772. Zoologiae Fundamenta, Praelectionibus Academicis Accommodata. Grunde i Dyrelaeren. Apud Frider. Christ. Pelt. Hafniae et Lipsiae. 254 p.

Burmeister H. 1861. Reise durch die La Plata-Staaten. Mit besonderer Rücksicht auf die physische Beschaffenheit und den Kulturzustand der Argentinischen Republik, ausgeführt in den Jahren 1857,1858, 1859 und 1860. Druck und Verlag von H. W. Schmidt. Halle. Zweiter Band. 538 p. 1 Karte.

Cabello C. 1978. La Nutria de Mar (Lutra felina) en la Isla de Chiloé, Chile. pp: 108-118. In N. Duplaix (Ed.). Otters: Proceedings of the Firts Working Meeting of the Otter Specialist Group. WWF / SSC / IUCN. Paramaribo, Suriname 27-29 March 1977. Internacional Union for Conservation of Nature and Natural Resources, Publication New Series. Morges, Switzerland. $158 \mathrm{p}$.

Cabello, C. 1983. La Nutria de Mar en la Isla de Chiloé. CONAF. Bol. Tec. $\mathrm{N}^{\circ} 6.37 \mathrm{p}$.

Cabrera A. 1957. Catálogo de los Mamíferos de América del Sur. Revista del Museo Argentino de Ciencias Naturales "Bernardino Rivadavia" e Instituto Nacional de Investigación de las Ciencias Naturales. Editora Coni. Ciencias Zoologicas, Tomo 4, $\mathrm{N}^{\circ} 1,308$ p. Buenos Aires.

Castilla J.C. \& I. Bahamondes. 1979. Observaciones conductuales y ecológicas sobre Lutra felina (Molina 1782) (Carnívora: Mustelidae) en las zonas central y centro-norte de Chile. Archivos de Biología y Medicina Experimentales, 12:119-132.

Castilla J.C. 1982. Nuevas observaciones sobre conducta, ecología y densidad de Lutra felina (Molina 1782) (Carnivora: Mustelidae) en Chile. Publicación Ocasional, Museo Nacional de Historia Natural 38: 197-206.

Chehébar C. 1990. Action Plan for Latin American Otters, pp: 64-73. In: P. Foster-Turley, S. Macdonald and C. Mason. Otters. An Action Plan for their Conservation. IUCN/SSC Otter Specialist Group. 126 p.

Clarke K.R. 1993. Non-parametric Multivariate Analyses of Changes in Community Structure. Australian Journal of Ecology 18 (1): 117-143. doi:10.1111/j.1442-9993.1993.tb00438.x.

CDC-UNALM. 2000. Ficha Tecnica, Gato Marino (Lutra felina). Amigo Guarda: Boletin para los Guardaparques del Peru. Centro de Datos para la Conservacion, Universidad Nacional Agraria La Molina. No 38:3-5. Lima.

Coker R.E. 1908. The Fisheries and the Guano Industry of Peru. Bulletin of the Bureau of Fisheries. Proceedings of the Fourth International Fishery Congress, 1908. Washington, U.S.A. Volume XXVIII, pp: 333-365.

Córdova, O., J. R. Rau, C. G. Suazo y A. Arriagada. 2009. Estudio comparativo de la ecología alimentaria del depredador de alto nivel trófico Lontra felina (Molina, 1782) (Carnivora: Mustelidae) en Chile. Revista de Biología Marina y Oceanografía 44(2): 429-438.
Coues E. 1877. Fur-bearing animals: a monograph of North American Mustelidae. Dep. Interior. Misc. Publ. $N^{\circ}$ 8. Govt. Print. Off. Washington. 348 p. 20 pl.

CPPS. 2004. III Reunión de expertos para revisar las actividades del Plan de Acción para la conservación de los mamíferos marinos del Pacífico Sudeste - Informe de la Reunión. Plan de Acción para la Protección del Medio Marino y Áreas Costeras del Pacífico Sudeste. Comisión Permanente del Pacífico Sur (CPPS), Programa de las Naciones Unidas para el Medio Ambiente (PNUMA). Marzo, Lima - Perú. 209 p. (Informe Inédito de la Reunión).

Delgado-Rodriguez C. 2005. Feeding ecology of Sea cat (Lontra felina) in southern Chile. River Otter Journal. 14(1) 1,2-7,8.

Dormann F., Carsten, J.M. McPherson, M.B. Araújo, et al. 2007. «Methods to Account for Spatial Autocorrelation in the Analysis of Species Distributional Data: a Review». Ecography 30 (5): 609-628. doi:10.1111/j.2007.09067590.05171.x.

Ebensperger L.A. \& J.C. Castilla. 1991. Conducta y densidad poblacional de Lutra felina en Isla Pan de Azúcar (III Región), Chile. Medio Ambiente 11:79-83.

Erxleben, J. C. P. 1777. Systema Regni Animalis per Classes, Ordines, Genera, Species, Varietates cum Synonymia et Historia Animalium. Classis I. Mammalia. Impensis Weygandianis, Lipsiae, $636 \mathrm{p}$.

FAO-ACMRR. 1978. Mammals in the Seas, Vol. 1. Report of the FAO Advisory Committee on Marine Resources Research. Working Party on Marine Mammals \& United Nations Environment Programme. FAO Fish. Ser. № 5, Rome. 264 p.

Gay C. 1847. Zoología, Tomo primero. En Historia física y política de Chile: según documentos adquiridos en esta República durante doce años de residencia en ella. París: en casa del autor. Chile: Museo de Historia Natural de Santiago. Tomo primero. $496 \mathrm{p}$.

Gervais F.L.P. 1841. Mammifères. pp: 1-68. In J. F. T. Eydoux and L. F. A. Souleyet (eds.). Zoologie. Voyage autour du Monde exécuté pendant les années 1836 et 1837 , sur la corvette La Bonite commandee par M. Vaillant. Arthus Bertrand, Editeur. Paris. Tome 1. 334 p. pl. 12.

Gray J.E. 1837. Description of some new o little knowm Mammalia, principally in the British Museum Collection. The Magazine of Natural History. New Series, Vol. 1. pp: 577-587. London.

Gray J.E. 1843. Descriptions of some new genera and species of Mammalia in the British Museum Collection. The Annals Magazine of Natural History. Printed and published by R. and J. E. Taylor. Vol. Xl (68): 81-160. London

Gray J.E. 1865. Revision of the Genera and Species of Mustelidae Contained in the British Museum. Proceedings of the Scientific Meetings of the Zoological Society of London. $906 \mathrm{p}$.

Goodwin, H. A. \& C. W. Holloway. 1978. Red Data Book, Vol 1 Mammalia, Survival Service Commission. 1972 New Edition, Updates from 1972-1978. Revised by Jane Thornback. Fauna Preservation Society, UNESCO, UNEP, Van Tienhoven Foundation, WWF \& Zoologische Gesellschaft. IUCN, Morges, Switzerland.

Grimwood, I. R. 1967. Recommendations on the Conservation of Wild Life and the Establishment of National Parks and Reserves in Peru. British Ministry of Overseas Development. June 1965 to March 1967. (unpublished report).

Grimwood, I. R. 1968a. Appendix III to Recommendations on the Conservation of Wild Life and the Establishment of National Parks and Reserves in Peru. British Ministry of Overseas Development. 100 p. +11 maps. (unpublished report). 
Grimwood, I. R. 1968b. Endangered Mammals in Peru. Oryx. 9(6): 411-421.

Grimwood, I. R. 1969. Notes on the distribution and status of some Peruvian mammals 1968. IUCN Commission on National Parks; American Committee for International Wild Life Protection; New York Zoological Society. Special publication $\mathrm{N}^{\circ} 21,86 \mathrm{p}$

Hammer Ø., Harper, D.A.T., \& P. D. Ryan, 2001. PAST: Paleontological Statistics Software Package for Education and Data Analysis. Palaeontologia Electronica 4(1): 9pp

Harris C.J. 1968. Otters: A Study of the Recent Lutrinae. Weidenfeld and Nicolson. London, United Kingdom.

ICZN. 1998. Opinion 1894. Regnum Animale ..., Ed. 2 (M.J. Brisson, 1762): rejected for nomenclatural purposes, with the conservation of the mammalian generic names Philander (Marsupialia), Pteropus (Chiroptera), Glis, Cuniculus and Hydrochoerus (Rodentia), Meles, Lutra and Hyaena (Carnivora), Tapirus (Perissodactyla), Tragulus and Giraffa (Artiodactyla). International Commission on Zoological Nomenclatura. Bulletin of Zoological Nomenclature 55(1): 64-71.

INEI. 2010. Perú: Anuario de Estadísticas Ambientales 2010. Instituto Nacional de Estadística e Informática, Dirección Técnica de Demografía e Indicadores Sociales. Lima-Perú. 523 p.

INGEMMET. 1995. Geología del Perú. Instituto Geologico Minero y Metalurgico. Serie A: Carta Geológica Nacional. Bol. 55. Lima. 177 p. +5 mapas.

INRENA. 1999. Resumen del Simposio Internacional sobre Conservación del Lobo de Río (Pteronura brasiliensis). Instituto Nacional de Recursos Naturales del Peru. Lima, 10-11 Setiembre 1998 (Informe Inedito).

Kerr R. 1792. The animal kingdom, or zoological system of the celebrated Sir Charles Linnaeus; Class I. Mammalia; containing a complete systematic description, arrangement, and nomenclature, of all the known species and varieties of the Mammalia, or animals which give suck to their young; being a translation of that part of the Systema Naturæ, as lately published, with great improvements, by Professor Gmelin of Goettingen. Together with numerous additions from more recent zoological writers, and illustrated with copperplates. Printed for J. Murray \& R. Faulder. 644 p. London.

Koepcke H-W. 1958. Introducción al estudio de la ecología y biogeografía: Con referencia especial al Perú. Serie de Divulgación Científica 11. Lima: Ministerio de Agricultura, Dirección de Pesquería y Caza.

Kostritsky L. 1963. Los mamíferos marinos de importancia económica. Recursos Naturales del Mar, 23, Servicio de Pesqueria del Ministerio de Agricultura. Facultad de Ciencias de la Universidad Nacional Mayor de San Marcos.

Larivière S. 1998. Lontra felina. Mammalian Species, American Society of Mammalogists. No. 575, pp. 1-5, 3 figs.

Larrañaga D.A. 1923. Escritos. Instituto Histórico y Geográfico del Uruguay. Botánica y Zoología. Tomo 2. Montevideo.

Legendre P. \& M.J. Fortin. 1989. Spatial Pattern and Ecological Analysis. Vegetatio 80 (2): 107-138.

Legendre P, Legendre LFJ (1998) Numerical ecology, 2nd edn. Elsevier Science, Amsterdam

Lesson R. P. 1842. Nouveau Tableau du Règne Animal: Mammifères. Arthus Bertrand, Paris. 204 p.

Ley No 28621. 2005. Ley de Líneas de Base del Dominio Marítimo del Perú. Diario Oficial El Peruano: Normas Legales. Año XXII, No 9272, pp: 303555-303564.

Linnaei C. 1758. Systema Naturae. Regnum Animale. Sistema Natur per Regna Tria Naturae secundum Classes, Ordines, Genera, Species, cum Characteribus, Differentiis, Synonymis, Locis. Tomus I. Editio Decima, Reformata. Holmiae: Impensis Direct Laurentii Salvia. 823 p.
Majluf P. \& Reyes J.C. 1989. The Marine Mammals of Peru: a review. In: Pauly D, P Muck, J Mendo and I Tsukayama (eds.) The Peruvian upwelling ecosystem: dynamics and interactions. ICLARM Conference Proceedings: 344-363.

Mangel, J. C., T. Whitty, G. Medina-Vogel, J. Alfaro-Shigueto, C. Caceres \& B. J. Godley. 2011. Latitudinal Variation in Diet and Patterns of Human Interaction in the Marine Otter. Marine Mammal Science, 27(2): E14-E25. DOI: 10.1111/j.1748-7692.2010.00414.x

Medina-Vogel, G. 1995a. Activity budget and social behaviour of Marine otter (Lutra felina) in Southern Chile. In: Reuther C \& D Rowe-Rowe (eds). Proceedings VI. International Otter Colloquium, Pietermaritzburg 1993. Hankensbüttel, Germany. Habitat, 11:62-64.

Medina-Vogel, G. 1995b. Feeding habits of Marine otter (Lutra felina) in Southern Chile. In: C Reuther \& D Rowe-Rowe (eds). Proceedings VI. International Otter Colloquium, Pietermaritzburg 1993. Hankensbüttel, Germany. Habitat, 11:65-68.

Medina-Vogel G., V.S. Kaufman, R. Monsalve \& V. Gomez. 2003. The influence of riparian vegetation, woody debris, stream morphology and human activity on the use of rivers by Southern river otters in Lontra provocax in Chile. Oryx 37: 422-430.

Medina, G., C. Delgado., Alvarez. R and Bartheld. J. 2004. Trophic Ecology of the Marine Otter (Lontra felina, Molina 1782) in Rocky Seashore of the South of Chile. Marine Mammal Science: Vol. 20, No. 1, pp. 134-144.

Medina-Vogel G. 2005. Estrategia regional para la conservación del huillìn (Lontra provocax) en Chile. Pp. 505-515, en: Smith-Ramirez C, JJ Armesto \& C Valdovinos (eds.), Historia, Biodiversidad y Ecología de los Bosques Costeros de Chile. Editorial Universitaria, Santiago, Chile. 708 pp.

Medina-Vogel, G., Bartheld, J. L., Pacheco, R. A. \& Delgado, R. 2006: Population Assessment and Habitat use by Marine Otter Lontra felina in Southern Chile. Wildlife. Biology. 12: 191-199.

Medina-Vogel G., F. Boher, G. Flores, A. Santibañez \& C. Soto-Azat. 2007. Spacing behavior of marine otters (Lontra felina) in relation to land refuges and fishery waste in Central Chile. Journal of Mammalogy 88(2): 487-494.

Medina-Vogel G., L.O. Merino, R. Monsalve-Alarco \& J.A. Vianna. 2008. Coastal-marine discontinuities, critical patch size and isolation: implications for marine otter conservation. Animal Conservation 11: 57-64.

Molina G.I. 1782. Saggio sulla storia naturale del Chili. Nella Stamperia di S. Tommaso d'Aquino. 368 p. map. Bologna

Nichols, J. D. \& B. K. Williams. 2006. Monitoring for conservation. Trends in Ecology \& Evolution 21 (12): 668-673.

Olavarría C. \& M. Sepúlveda. 1995. Ecología del Chungungo Lutra felina (Molina, 1782) en el Monumento Natural Isla Cachagua. Boletín Técnico 61, CONAF, V Región, Chile. 19 p.

Osgood W.H. 1943. The Mammals of Chile. Field Museum of Natural History. Zoological Series. Chicago U.S.A. Vol. 30. 268 p.

Parera, A. 1996. Las nutrias verdaderas de la Argentina. Boletín Técnico $N^{\circ} 21$. Fundación Vida Silvestre Argentina, Buenos Aires, $31 \mathrm{p}$.

Pennant T. 1793. History of Quadrupeds. Third Edition. B \& J. White, Vol. 2. London. 324 p.

Pizarro J. 2008. Mortality Of The Marine Otter (Lontra felina) In Southern Peru. IUCN Otter Spec. Group Bull. 25(2):94-99

Pohle, H. 1920. Die Unterfamilie der Lutrinae. (Eine systematischtiergeographische Studie an dem Material der Berliner Museen). Archiv für Naturgeschichte. Jahrg. 85, Abt. A. heft 9.247 p. (REFERENCIA NO REVISADA).

Pulido V. 1991. El Libro Rojo de la Fauna Silvestre del Perú. Instituto Nacional de Investigación Agraria y Agroindustrial, World Wildlife Fund U.S. y U.S. Fish and Wildlife Service. Lima, 219 p. 
Quijada B.B. 1910. Catálogo ilustrado i descriptivo de la colección de mamíferos vivientes. Boletín del Museo Nacional de Chile Tomo I N. ${ }^{\circ} 8$.

Reyes J.C. 1992. Informe Nacional sobre la Situación de los Mamíferos Marinos en Perú. Comisión Permanente del Pacífico Sur. Informes y Estudios del Programa de Mares Regionales del PNUMA No. 145. CPPS/PNUMA. 21 p.

Rodrigues, A. S. L., J. D. Pilgrim, J. F. Lamoreux, M. Hoffmann \& T. M. Brooks. 2006. The value of the IUCN Red List for conservation. Trends in Ecology \& Evolution 21 (2) (febrero 1): 71-76.

Rozzi, R. \& J.C. Torres-Mura. 1990. Observaciones del chungungo (Lutra felina) al sur de la Isla Grande de Chiloé: antecedentes para su conservación. Medio Ambiente 11(1): 24-28

Sánchez, R. 1990. Observaciones Ecológicas en el Gato Marino Lutra felina (Mol.), Isla San Gallan, Reserva Nacional de Paracas. Resumen. pp: 65. En: Centro de Investigaciones sobre Mamíferos Marinos (CIMMA). 4ta Reunión de Trabajo de especialistas en Mamíferos Acuáticos de América del Sur. 12-15 Nov. Valdivia-Chile. (Resumen).

Sánchez R. 1992. Distribución y Abundancia del Gato Marino Lutra felina en el Departamento de Ica. Programa de Subvenciones WCI/APECO. 29 p. (Informe Inedito).

Santillán L. \& K. Caro. 2007. Mamíferos Marinos en el Puerto de Salaverry y Zonas Adyacentes. Libro de resúmenes CONCIMAR 2007.

Schinz, H. R. 1844. Systematisches Verzeichniß aller bis jetzt bekannten Säugethiere oder Synopsis mammalium nach dem Cuvier'schen System. Erster Band. Solothurn. 587 p.

Schweigger E. 1964. El litoral peruano. Gráfica Morsom S. A., Lima, $414 p$.

Schweigger, E. 1947. El Litoral Peruano. Compañía Administradora del Guano. Lima, Perú. 262 p.

Sexton, J. P., P. J. McIntyre, A. L. Angert \& K. J. Rice. 2009. Evolution and Ecology of Species Range Limits. Annual Review of Ecology, Evolution, and Systematics 40 (1) (diciembre): 415-436.

Shaw G. 1800. General zoology or systematic natural history. Mammalia. Vol. I. Part 2. London. Printed for G. Kearsley. pp: 249-552.

Sielfeld, W. K. 1990a. Dieta del Chungungo (Lutra felina (Molina, 1782)) (Mustelidae, Carnivora) en Chile Austral. Investigaciones en Ciencia y Tecnología, Serie: Ciencias del Mar 1: 23-29.

Sielfeld W. 1992. Abundancias relativas de Lutra felina (Molina, 1782) y L. provocax Thomas, 1908 en el litoral de Chile Austral. Investigaciones Científicas y Técnicas, Serie Ciencias del Mar (Chile) 2: 3-12.

Sielfeld W. 1997. Las áreas protegidas de la XII Región de Chile en la perspectiva de los mamíferos marinos. Estudios Oceanológicos, 16:87-107.

Sielfeld W. \& J.C. Castilla. 1999. Estado de conservación y conocimiento de las nutrias en Chile. Estud. Oceanol. 18:69-79.

Sinclair, A. R. E., J. M. Fryxell, \& G. Caughley. 2009. Wildlife Ecology, Conservation and Management. Wiley.

Sokal R.R. \& N.L. Oden. 1978. Spatial Autocorrelation in Biology: 1. Methodology. Biological Journal of the Linnean Society 10 (2): 199-228. doi:10.1111/j.1095-8312.1978.tb00013.x.

Taylor, B. L., M. Martinez, T. Gerrodette, J. Barlow, y Y. N. Hrovat. 2007. Lessons from Monitoring Trends in Abundance of Marine Mammals. Marine Mammal Science 23 (1): $157-175$.

Thomas O. 1889. Preliminary Notes on the Characters and Synonymy of the different Species of Otter. Proceedings of the Scientific Meetings of the Zoological Society of London. pp: 190-200.
Thomas O. 1908. On certain African and South American Otters. The Annals Magazine of Natural History. Printed and Published by Taylor and Francis. Vol. 1. Ser. 8. pp: $387-$ 395. London

Thornback, J. and Jenkins, M. 1982. The IUCN Mammal Red Data Book. Part 1: Threatened Mammalian taxa of the Americas and the Australasian zoogeographic region (excluding Cetacea). IUCN, Gland, Switzerland. $516 \mathrm{p}$.

Tomes R.F. 1861. Report of a Collection of Mammals made by Osbert Salvin, Esq., E.Z.S., at Dueñas, Guatemala; with Notes on some of the Species, by Mr. Fraser. Proceedings of the Scientific Meetings of the Zoological Society of London. pp: 278-288.

Tschudi J.J. von. 1844. Untersuchungen über die Fauna Peruana. Therologie. Druck und Verlag von Scheitlin und Zollikofer. St. Gallen. 262 p.

Tschudi, J.J. von. 1846. Peru: Reiseskizzen aus den jahren 1838 1842. Erster Band. St. Gallen. Verlag von Scheitlin und Zollikofer. $346 \mathrm{p}$.

UICN. 2001. Categorías y Criterios de la Lista Roja de la UICN: Versión 3.1. Comisión de Supervivencia de Especies de la UICN. UICN, Gland, Suiza y Cambridge, Reino Unido. 33 p.

Valqui J., G.B. Hartl \& F.E. Zachos. 2010. Non-invasive Genetic Analysis Reveals High Levels of mtDNA Variability in the Endangered South-American Marine Otter (Lontra Felina). Conservation Genetics 11 (5): 2067-2072. doi:10.1007/ s10592-010-0069-9.

Valqui, J. 2012. The Marine Otter Lontra felina (Molina, 1782): A Review of Its Present Status and Implications for Future Conservation. Mammalian Biology, 77:75-83

van Zyll de Jong, C.G. 1972. A systematic review of the Nearctic and Neotropical river otters (genus Lutra, Mustelidae, Carnivora). Royal Ontario Museum, Life Sciences Contribution, 80:1-104.

Vaz-Ferreira R. 1979. Situación poblacional y conservación de los mamíferos marinos en Latinoamérica. Act Zool. Lilloana 34: 91-101.

Vianna J.A, P. Ayerdi, G. Medina-Vogel, et al. 2010. Phylogeography of the Marine Otter (Lontra felina): historical and contemporary factors determining its distribution. The Journal of heredity 101 (6): 676-689. doi:10.1093/jhered/esq088.

Vidal O. 1992. Los mamíferos del Océano Pacífico Sudeste (Panamá, Colombia, Ecuador, Perú y Chile): Diagnóstico regional. Informe y Estudios del Programa de Mares Regionales del PNUMA N ${ }^{\circ}$. 142. CPPS/PNUMA. 26 p.

Wagner J.A. 1841. Die Säugethiere in Abbildungen nach der Natur. Die Säugthiere in Abbildungen nach der Natur, mit Beschreibungen von Dr. Johann Christian Daniel von Schreber. Supplementband, Abth. 2. 558 pp. Leipzig.

Waterhouse G.R. 1839. Mammalia Part 2. In C. Darwin (Ed.). The zoology of the voyage of HMS Beagle, during the years 1832 to 1836. London. Smith Elder and Co. pl. 35, 97 p.

Wozencraft W.C. 2005. Order Carnivora. pp: $532-628$. In D. E. Wilson \& D. M. Reeder (eds.). Mammal Species of the World. A Taxonomic and Geographic Reference (3rd ed). The Johns Hopkins University Press. Vol. 1. 740 p. Baltimore. 
Apéndice 1. Sinopsis de la historia taxonómica de la nutria marina, desde la determinación de la especie tipo Mustela lutra hasta el actual nombre científico Lontra felina. Se incluye la localidad tipo o distribución mencionada en el documento citado. También se han adicionado otras identificaciones que explican el origen de algunos sinónimos o clasificaciones relacionadas con el género Lutra o Lontra.

\begin{tabular}{|c|c|c|c|c|c|c|c|c|}
\hline \multirow{3}{*}{\begin{tabular}{l|} 
Año \\
1758 \\
\end{tabular}} & \multirow{3}{*}{\begin{tabular}{|l|} 
Autor \\
Linneo
\end{tabular}} & \multicolumn{2}{|c|}{ Determinación o Referencia } & \multirow[t]{2}{*}{ Localidad tipo o Distribución } & \multicolumn{2}{|r|}{ Sinónimo } & \multirow{2}{*}{\multicolumn{2}{|c|}{ Páginas }} \\
\hline & & \multirow{2}{*}{$\begin{array}{l}\text { Género } \\
\text { Mustela }\end{array}$} & \multirow{2}{*}{$\begin{array}{l}\text { Especie } \\
\text { lutra }\end{array}$} & & \multirow[t]{2}{*}{ Género } & Especie & & \\
\hline & & & & & & & 45 & T.1 \\
\hline 1762 & Brisson & Lutra & & & & & $201-03$ & \\
\hline 1772 & Brünnichii & Lutra & & & & & 42 & \\
\hline 1782 & Molina & Mustela & felina & Chili (Chile) & & & $284-85,34$ & \\
\hline 1792 & Kerr & Mustela (Lutra) & chilensis & Costa de Chili (Chile) & & & 172 & \\
\hline 1800 & Bechstein & Lutra & felina & Chili (Chile) & & & 406,695 & V. 2 \\
\hline 1800 & Shaw & Lutra & felina & Chili (Chile) & & & $448-49$ & V.1 P.2 \\
\hline 1832 & Bennet & Lutra & chilensis & Chili (Chile) & & & $1-2$ & P. 2 \\
\hline 1837 & Gray & Lutra & californica & California & & & 580 & V.1 \\
\hline \multirow[t]{2}{*}{1839} & \multirow[t]{2}{*}{ Waterhouse } & Lutra & platensis & La Plata (Maldonado, Uruguay) & & & $21-22$ & \\
\hline & & Lutra & chilensis & Archipiélago de Chonos (Chile) & & & $22-24$ & \\
\hline 1841 & Wagner & Lutra & brachydactyla & América Occidental & & & $260-61$ & \\
\hline 1841 & Gervais & Lutra & peruviensis & Isla San Lorenzo (Perú) & & & $15-17$ & T. 1 \\
\hline \multirow[t]{2}{*}{1842} & \multirow[t]{2}{*}{ Lesson } & Lutra & chilensis & Archipiélago de Chonos (Chile) & $\begin{array}{l}\text { Lutra } \\
\text { Mustela }\end{array}$ & $\begin{array}{l}\text { felina (Shaw, 1800) } \\
\text { felina (Molina, 1782) }\end{array}$ & 73 & \\
\hline & & Lutra & peruviensis & Perú (San Lorenzo) & & & 73 & \\
\hline 1843 & Gray & Lontra & & & & & 118 & V. 11 \\
\hline \multirow[t]{2}{*}{1844} & \multirow[t]{2}{*}{ Schinz } & Lutra & chilensis & Chile, Archipiélago de Chonos. & & & 352 & V.1 \\
\hline & & Lutra & felina & Chili (Chile) & & & $355-56$ & V.1 \\
\hline \multirow[t]{2}{*}{1844} & \multirow[t]{2}{*}{ Tschudi } & Lutra & chilensis & $\begin{array}{l}\text { Toda la costa occidental de Sudamérica hasta } \\
\text { el sur de California (USA). Observado en el } \\
\text { archipiélago de Lemos ( } 45^{\circ} 12 \text { 'S), Isla de Chiloé } \\
\text { y el Callao. Referenciado en Cobija, Iquique, } \\
\text { Trujillo y Ecuador }\end{array}$ & Lutra & peruviensis (Gervais, 1841) & 119 & \\
\hline & & Lutra & montana & $\begin{array}{l}\text { Comas y Andamarca (Junín), Huanta } \\
\text { (Ayacucho) - Perú }\end{array}$ & & & 120 & \\
\hline 1847 & Gay & Lutra & felina & $\begin{array}{l}\text { Isla de Chiloé, Arch. de Chonos, Isla del Fuego } \\
\text { (Argentina-Chile) }\end{array}$ & $\begin{array}{l}\text { Mustela } \\
\text { Lutra }\end{array}$ & $\begin{array}{l}\text { felina (Molina, 1782) } \\
\text { chilensis (Bennet, 1832) }\end{array}$ & $45-46$ & T. 1 \\
\hline 1855 & Baird & Lutra & felina & Costa de Chile & $\begin{array}{l}\text { Mustela } \\
\text { Lutra } \\
\text { Lutra } \\
\end{array}$ & $\begin{array}{l}\text { felina (Molina, 1782) } \\
\text { chilensis (Bennet, 1832) } \\
\text { felina (Gay, 1847) }\end{array}$ & 165 & V. 2 \\
\hline 1861 & Burmeister & Lutra & paranensis & $\begin{array}{l}\text { Río Paraná (Paraguay). Río Salado, río Dulce y } \\
\text { laguna de los Porongos (Argentina). }\end{array}$ & & & $410-11$ & V. 2 \\
\hline 1865 & Gray & Nutria & felina & $\begin{array}{l}\text { Chiloé, Chili (Chile), Perú, California (USA), } \\
\text { Kamschatka (Rusia). }\end{array}$ & $\begin{array}{l}\text { Lutra } \\
\text { Lutra } \\
\text { Lutra } \\
\text { Lutra }\end{array}$ & $\begin{array}{l}\text { felina (Shaw, 1800) } \\
\text { chilensis (Bennet, 1832) } \\
\text { californica (Gray, 1837) } \\
\text { platensis (Waterhouse, 1839) }\end{array}$ & $128-29$ & \\
\hline 1877 & Coues & Lutra & californica & De Chili (Chile) a Kamschatka (Rusia) & $\begin{array}{l}\text { Mustela } \\
\text { Lutra } \\
\text { Lutra } \\
\text { Lutra } \\
\text { Nutria } \\
\end{array}$ & $\begin{array}{l}\text { felina (Molina, 1782) } \\
\text { chilensis (Bennet, 1832) } \\
\text { californica (Gray, 1837) } \\
\text { platensis (Waterhouse, 1839) } \\
\text { felina (Gray, 1865) }\end{array}$ & 301 & \\
\hline $1879-82$ & Alston & Lutra & felina & $\begin{array}{l}\text { Norte América, vertiente del Pacífico desde } \\
\text { Alaska? México, Guatemala, Costa Rica, } \\
\text { Panamá, Sudamérica hasta Chile }\end{array}$ & $\begin{array}{l}\text { Mustela } \\
\text { Lutra } \\
\text { Lutra } \\
\text { Nutria } \\
\text { Lutra }\end{array}$ & $\begin{array}{l}\text { felina (Molina, 1782) } \\
\text { chilensis (Bennet, 1832) } \\
\text { californica (Gray, 1837) } \\
\text { felina (Gray, 1865) } \\
\text { felina (Coues, 1877) }\end{array}$ & $86-87$ & \\
\hline 1889 & Thomas & Lutra & felina & $\begin{array}{l}\text { Estrecho de Magallanes, Patagonia, Chile, Perú y } \\
\text { Ecuador (San Lorenzo) }\end{array}$ & \begin{tabular}{|l|} 
Mustela \\
Mustela (Lu.) \\
Lutra \\
Lutra \\
Lutra \\
Lutra \\
Lutra \\
Nutria \\
\end{tabular} & $\begin{array}{l}\text { felina (Molina, 1782) } \\
\text { chilensis (Kerr, 1792) } \\
\text { felina (Shaw, 1800) } \\
\text { chilensis (Bennet, 1832) } \\
\text { californica (Gray, 1837) } \\
\text { peruviensis (Gervais, 1841) } \\
\text { brachydactyla (Wagner, 1841) } \\
\text { felina (Gray, 1865) }\end{array}$ & $197-99$ & \\
\hline 1905 & Allen & Lutra & felina & $\begin{array}{l}\text { Costa de Chile, Estrecho de Magallanes, Tierra } \\
\text { de Fuego (Chile y Argentina) }\end{array}$ & $\begin{array}{l}\text { Mustela } \\
\text { Mustela (Lu.) } \\
\text { Lutra } \\
\text { Lutra }\end{array}$ & $\begin{array}{l}\text { felina (Molina, 1782) } \\
\text { chilensis (Kerr, 1792) } \\
\text { felina (Shaw, 1800) } \\
\text { chilensis (Bennet, 1832) }\end{array}$ & $148-50$ & V.3 \\
\hline
\end{tabular}

(Continúa...) 
Apéndice 1. Continuación.

\begin{tabular}{|c|c|c|c|c|c|c|c|c|}
\hline \multirow{3}{*}{ Año } & \multirow{3}{*}{ Autor } & \multicolumn{2}{|c|}{ Determinación o Referencia } & \multirow[t]{2}{*}{ Localidad tipo o Distribución } & \multicolumn{2}{|r|}{ Sinónimo } & \multirow{2}{*}{\multicolumn{2}{|c|}{ Páginas }} \\
\hline & & Género & Especie & & Género & Especie & & \\
\hline & & & & & $\begin{array}{l}\text { Lutra } \\
\text { Lutra } \\
\text { Lutra } \\
\text { Nutria }\end{array}$ & $\begin{array}{l}\text { californica (Gray, 1837) } \\
\text { peruviensis (Gervais, 1841) } \\
\text { brachydactyla (Wagner, 1841) } \\
\text { felina (Gray, 1865) }\end{array}$ & & \\
\hline 1908 & Thomas & Lutra & peruviensis & Isla San Lorenzo (Perú) & Lutra & cinerea (Thomas, 1908) & 393 & V.1S.8 \\
\hline 1920 & Pohle & Lutra & peruensis & & & & & \\
\hline 1923 & Larrañaga & Lutra & lutris & & & & 341,345 & T. 2 \\
\hline 1943 & Osgood & Lutra & felina & Costa de Chile hasta el norte de Perú & $\begin{array}{l}\text { Mustela } \\
\text { Mustela (Lu.) } \\
\text { Lutra } \\
\text { Lutra } \\
\text { Lutra } \\
\end{array}$ & $\begin{array}{l}\text { felina (Molina, 1782) } \\
\text { chilensis (Kerr, 1792) } \\
\text { chilensis (Bennet, 1832) } \\
\text { californica (Gray, 1837) } \\
\text { brachydactyla (Wagner, 1841) }\end{array}$ & $90-91$ & V. 30 \\
\hline 1957 & Cabrera & Lutra & felina & $\begin{array}{l}\text { Costa sudamericana del Pacífico, desde } \\
\text { Perú hasta el estrecho de Lemaire, y las islas } \\
\text { inmediatas (Perú, Chile y extremo sur de } \\
\text { Argentina) }\end{array}$ & $\begin{array}{l}\text { Mustela } \\
\text { Mustela (Lu.) } \\
\text { Lutra } \\
\text { Lutra } \\
\text { Lutra } \\
\text { Lutra } \\
\text { Lutra } \\
\text { Nutria } \\
\text { Lutra } \\
\end{array}$ & $\begin{array}{l}\text { felina (Molina, 1782) } \\
\text { chilensis (Kerr, 1792) } \\
\text { felina (Bechstein, 1800) } \\
\text { chilensis (Bennet, 1832) } \\
\text { californica (Gray, 1837) } \\
\text { peruviensis (Gervais, 1841) } \\
\text { brachydactyla (Wagner, 1841) } \\
\text { felina (Gray, 1865) } \\
\text { cinerea? (Thomas, 1908) }\end{array}$ & $272-73$ & \\
\hline 1968 & Harris & Lutra & felina & $\begin{array}{l}\text { Costa occidental de Sudamérica, desde Ecuador } \\
\text { (?) por el norte hasta el estrecho de Lemaire por } \\
\text { el sur, además de las islas costeras. }\end{array}$ & $\begin{array}{l}\text { Mustela } \\
\text { Mustela (Lu.) } \\
\text { Lutra } \\
\text { Lutra } \\
\text { Lutra } \\
\text { Lutra } \\
\text { Lutra } \\
\text { Lutra } \\
\text { Lutra } \\
\text { Nutria } \\
\text { Lutra } \\
\text { Lutra } \\
\text { Lutra }\end{array}$ & $\begin{array}{l}\text { felina (Molina, 1782) } \\
\text { chilensis (Kerr, 1792) } \\
\text { chilensis (Bennet, 1832) } \\
\text { californica (Gray, 1837) } \\
\text { chilensis (Waterhouse, 1839) } \\
\text { peruviensis (Gervais, 1841) } \\
\text { brachydactyla (Wagner, 1841) } \\
\text { californix (Lesson, 1842) } \\
\text { chilensis (Schinz, 1844) } \\
\text { felina (Gray, 1865) } \\
\text { platensis (Gray, 1865, 1869) } \\
\text { cinerea? (Thomas, 1908) } \\
\text { felina (Pohle, 1920) }\end{array}$ & $216-17$ & \\
\hline 1972 & \begin{tabular}{|l} 
van Zyll de \\
Jong
\end{tabular} & Lontra & felina & $\begin{array}{l}\text { Costa del Pacífico desde el norte del Perú hasta } \\
\text { Cabo de Hornos (Nota: referido a Perú, Chile y } \\
\text { extremo sur de Argentina). }\end{array}$ & $\begin{array}{l}\text { Mustela } \\
\text { Mustela (Lu.) } \\
\text { Lutra } \\
\text { Lutra } \\
\text { Lutra } \\
\text { Lutra } \\
\text { Nutria } \\
\text { Lutra } \\
\text { Lutra } \\
\end{array}$ & $\begin{array}{l}\text { felina (Molina, 1782) } \\
\text { chilensis (Kerr, 1792) } \\
\text { chilensis (Bennet, 1832) } \\
\text { californica (Gray, 1837) } \\
\text { peruviensis (Gervais, 1841) } \\
\text { brachydactyla (Wagner, 1841) } \\
\text { felina (Gray, 1865) } \\
\text { felina (Pohle, 1920) } \\
\text { felina (Cabrera, 1958) }\end{array}$ & 88 & \\
\hline 1998 & Lariviere & Lontra & felina & $\begin{array}{l}\text { Costa del Pacífico, desde el norte de Perú (al } \\
\text { menos hasta Chimbote, límite norte a 9S) } \\
\text { hasta cabo de Hornos, Estrecho de Lemaire e } \\
\text { islas adyacentes. Estrecho de Magallanes e isla } \\
\text { de los Estados (Perú, Chile y extremo sur de } \\
\text { Argentina) }\end{array}$ & $\begin{array}{l}\text { Mustela } \\
\text { Mustela (Lu.) } \\
\text { Lutra } \\
\text { Lutra } \\
\text { Lutra } \\
\text { Lutra }\end{array}$ & $\begin{array}{l}\text { felina (Molina, 1782) } \\
\text { chilensis (Kerr, 1792) } \\
\text { californica (Gray, 1837) } \\
\text { brachydactyla (Wagner, 1841) } \\
\text { peruviensis (Gervais, 1841) } \\
\text { cinerea (Thomas, 1908) }\end{array}$ & $1-5$ & \\
\hline 2005 & Wozencraft & Lontra & felina & Extremo sur de Argentina, Chile y Perú & $\begin{array}{l}\text { Mustela (Lu.) } \\
\text { Lutra } \\
\text { Lutra } \\
\text { Lutra } \\
\text { Lutra } \\
\text { Lutra } \\
\text { Lutra } \\
\text { Lutra } \\
\text { Lutra }\end{array}$ & $\begin{array}{l}\text { chilensis (Kerr, 1792) } \\
\text { brachydactyla (Wagner, 1841) } \\
\text { californica (Gray, 1837) } \\
\text { peruviensis (Gervais, 1841) } \\
\text { montana (Tschudi, 1844) } \\
\text { paranensis (Burmeister 1861) } \\
\text { cinerea (Thomas, 1908) } \\
\text { peruensis (Pohle, 1920) } \\
\text { lutris (Larrañaga, 1923) }\end{array}$ & 603 & V.1 \\
\hline
\end{tabular}


Apéndice 2. Registros de individuos (Reg.) de Lontra felina en la costa del Perú entre los años 2000 y 2010.

\begin{tabular}{|c|c|c|c|c|c|c|}
\hline $\mathbf{N}^{\circ}$ & Dpto. & Localidad & Lat. S & Long. W & Reg. & Año \\
\hline 1 & Ancash & Pto. de Santa & $08^{\circ} 59^{\prime} 15,63^{\prime \prime}$ & " 78³9'22,81" & 2 & 2009 \\
\hline 2 & Ancash & Vesique & $09^{\circ} 12^{\prime} 45,90^{\prime \prime}$ & " 78²9'08,74" & 2 & 2003 \\
\hline 3 & Ancash & Is. Los Chimús & $09^{\circ} 21^{\prime} 02,42^{\prime \prime}$ & " $78^{\circ} 27^{\prime} 48,43^{\prime \prime}$ & 4 & 2003 \\
\hline 4 & Ancash & Tortugas & $09^{\circ} 22^{\prime} 10,75^{\prime \prime}$ & " 78²5'10,00" & 2 & 2000 \\
\hline 5 & Ancash & Pto. Casma & $09^{\circ} 27^{\prime} 22,54^{\prime \prime}$ & " 78²3'07,04" & 1 & 2010 \\
\hline 6 & Ancash & Pta. El Huaro & $09^{\circ} 37^{\prime} 38,09^{\prime \prime}$ & " 78²1'57,55" & 3 & 2010 \\
\hline 7 & Ancash & Playa Patillos & $09^{\circ} 53^{\prime} 12,07^{\prime \prime}$ & " 78¹4'02,36" & 4 & 2003 \\
\hline 8 & Ancash & Caleta Culebras & $09^{\circ} 56 ' 56,45^{\prime \prime}$ & " 78¹3'43,29" & 1 & 2010 \\
\hline 9 & Ancash & Pta. Culebras & $09^{\circ} 56 ' 59,91^{\prime \prime}$ & " 78¹4'01,54" & 2 & 2003 \\
\hline 10 & Ancash & El Erizo & $09^{\circ} 59^{\prime} 40,49^{\prime \prime}$ & " 78¹1'47,97" & 2 & 2008 \\
\hline 11 & Ancash & Pto. Huarmey & $10^{\circ} 05^{\prime} 50,34^{\prime \prime}$ & " 78¹0'16,59" & 2 & 2002 \\
\hline 12 & Ancash & Pta. Lagarto & $10^{\circ} 06^{\prime} 29,45^{\prime \prime}$ & " 78¹1'04,84" & 2 & 2010 \\
\hline 13 & Ancash & Pta. Colorada & $10^{\circ} 29 ' 31,32^{\prime \prime}$ & " 7757'53,49" & 1 & 2003 \\
\hline 14 & Lima & Pta. Litera & $10^{\circ} 36^{\prime} 44,29^{\prime \prime}$ & " 7753'08,01" & 1 & 2003 \\
\hline 15 & Lima & Huacho & $11^{\circ} 07^{\prime} 18,62^{\prime \prime}$ & " 77³7'06,89" & 1 & 2004 \\
\hline 16 & Lima & Pta. Salinas & $11^{\circ} 17^{\prime} 29,18^{\prime \prime}$ & " 77³9'08,83" & 6 & 2003 \\
\hline 17 & Lima & Pta. Chancay & $11^{\circ} 35^{\prime} 08,87^{\prime \prime}$ & " 77º'16'48,91" & 1 & 2009 \\
\hline 18 & Lima & Pta. San Francisco & $11^{\circ} 46^{\prime} 05,67^{\prime \prime}$ & " 77011'27,59" & 3 & 2002 \\
\hline 19 & Lima & Pta. Mulato & $11^{\circ} 46^{\prime} 07,12^{\prime \prime}$ & " 77¹1'50,65" & 2 & 2004 \\
\hline 20 & Lima & Playa Santa Rosa & $11^{\circ} 48^{\prime} 11,66^{\prime \prime}$ & " 77º'10'42,80" & 1 & 2005 \\
\hline 21 & Lima & Cabezo (Is. San Lorenzo) & $12^{\circ} 03^{\prime} 56,92^{\prime \prime}$ & " 77º14'57,90" & 1 & 2009 \\
\hline 22 & Lima & Las Peñas (Is. San Lorenzo) & $12^{\circ} 05^{\prime} 27,16^{\prime \prime}$ & " 77¹4'17,97" & 2 & 2008 \\
\hline 23 & Lima & Playa Regatas & $12^{\circ} 09^{\prime} 56,54^{\prime \prime}$ & " 7702'05,08" & 1 & 2004 \\
\hline 24 & Lima & Is. Pachacámac & $12^{\circ} 17^{\prime} 59,28^{\prime \prime}$ & " 76º54'08,74" & 2 & 2003 \\
\hline 25 & Lima & San Bártolo & $12^{\circ} 23^{\prime} 26,91^{\prime \prime}$ & " 76º $47101,59 "$ & 1 & 2002 \\
\hline 26 & Lima & Curayacu & $12^{\circ} 23^{\prime} 40,55^{\prime \prime}$ & " 76º 46 '49,33" & 1 & 2007 \\
\hline 27 & Lima & La Tiza & $12^{\circ} 24^{\prime} 52,82^{\prime \prime}$ & " 76º $4648,95^{\prime \prime}$ & 1 & 2002 \\
\hline 28 & Lima & Pta Boquerón & $12^{\circ} 26 \prime 39,25^{\prime \prime}$ & " 76 $47^{\circ} 03,18^{\prime \prime}$ & 1 & 2002 \\
\hline 29 & Lima & Playa el Carbón & $12^{\circ} 27^{\prime} 06,53^{\prime \prime}$ & " 76º $4659,01^{\prime \prime}$ & 1 & 2003 \\
\hline 30 & Lima & Naplo & $12^{\circ} 28^{\prime} 18,03^{\prime \prime}$ & " 76º $47^{\prime} 27,54^{\prime \prime}$ & 2 & 2002 \\
\hline 31 & Lima & Boquerón & $12^{\circ} 28^{\prime} 45,15^{\prime \prime}$ & " $76^{\circ} 48^{\prime} 04,18^{\prime \prime}$ & 1 & 2004 \\
\hline 32 & Lima & Pucusana & $12^{\circ} 28^{\prime} 47,84^{\prime \prime}$ & " 76º $47^{\prime} 59,06^{\prime \prime}$ & 3 & 2002 \\
\hline 33 & Lima & Cerros Lapa Lapa & $12^{\circ} 33^{\prime} 02,32^{\prime \prime}$ & " 76º 43 '57,11" & 2 & 2002 \\
\hline 34 & Lima & Pta. Cordero & $12^{\circ} 35^{\prime} 38,74^{\prime \prime}$ & " 76º $42^{\prime} 17,42^{\prime \prime}$ & 1 & 2002 \\
\hline 35 & Lima & Pto. Viejo & $12^{\circ} 35^{\prime} 22,52^{\prime \prime}$ & " 76º $42 ' 22,05^{\prime \prime}$ & 1 & 2008 \\
\hline 36 & Lima & Playa San Pablo & $12^{\circ} 35^{\prime} 58,45^{\prime \prime}$ & " 76º $41 ' 52,43^{\prime \prime}$ & 1 & 2008 \\
\hline 37 & Lima & León Dormido & $12^{\circ} 37^{\prime} 57,12^{\prime \prime}$ & " 76º' $40^{\prime} 17,39^{\prime \prime}$ & 1 & 2008 \\
\hline 38 & Lima & La Ensenada & $12^{\circ} 38^{\prime} 58,02^{\prime \prime}$ & " 76º'40'20,67" & 1 & 2002 \\
\hline 39 & Lima & Bujama & $12^{\circ} 44^{\prime} 11,81^{\prime \prime}$ & " 76º38'10,88" & 1 & 2002 \\
\hline 40 & Lima & Is. Asia & $12^{\circ} 47^{\prime} 17,29^{\prime \prime}$ & " 76º37'13,21" & 1 & 2003 \\
\hline 41 & Lima & Pasamayito & $12^{\circ} 49^{\prime} 15,03^{\prime \prime}$ & " 76³3'30,85" & 2 & 2008 \\
\hline 42 & Lima & Los Leones & $12^{\circ} 53^{\prime} 12,56^{\prime \prime}$ & " 76º30'56,33" & 1 & 2004 \\
\hline 43 & Lima & Pto. Fiel & $12^{\circ} 56 ' 41,78^{\prime \prime}$ & " 76º30'19,29" & 2 & 2004 \\
\hline 44 & Lima & Pta. Corrientes & $12^{\circ} 57^{\prime} 16,67^{\prime \prime}$ & " 76º30'53,53" & 2 & 2010 \\
\hline 45 & Lima & Pta. Honda & $12^{\circ} 58 ' 31,05^{\prime \prime}$ & " 76º'30'23,39" & 1 & 2008 \\
\hline 46 & Lima & Pta. Lobos & $12^{\circ} 58^{\prime} 57,42^{\prime \prime}$ & " 76º30'18,69" & 2 & 2007 \\
\hline 47 & Lima & Cerro Azul & $13^{\circ} 01^{\prime} 41,54^{\prime \prime}$ & " 76²9'16,80" & 3 & 2005 \\
\hline 48 & Lima & Río Cañete & $13^{\circ} 07^{\prime} 13,91^{\prime \prime}$ & " $76^{\circ} 23^{\prime} 11,10^{\prime \prime}$ & 1 & 2003 \\
\hline 49 & Ica & Playa Talpo & $13^{\circ} 48^{\prime} 05,27^{\prime \prime}$ & " 76²0'22,94" & 2 & 2006 \\
\hline 50 & Ica & Is. San Gallán & $13^{\circ} 50^{\prime} 12,43^{\prime \prime}$ & " 76²6'03,73" & 2 & 2002 \\
\hline 51 & Ica & Pta. Culebra & $13^{\circ} 50$ '38,54" & " 76º23'06,85" & 1 & 2003 \\
\hline 52 & Ica & Lagunilla & $13^{\circ} 53^{\prime} 52,02^{\prime \prime}$ & " 76º'18'38,11" & 3 & 2002 \\
\hline 53 & Ica & Yumaque & $13^{\circ} 54^{\prime} 23,79^{\prime \prime}$ & " 76º'17'27,29" & 2 & 2002 \\
\hline 54 & Ica & La Mina & $13^{\circ} 54^{\prime} 44,70^{\prime \prime}$ & " 76²19'05,91" & 2 & 2005 \\
\hline 55 & Ica & Playa Esperanza & $13^{\circ} 54^{\prime} 53,43^{\prime \prime}$ & " 76º $18 ' 57,94 "$ & 3 & 2001 \\
\hline 56 & Ica & Pta. Arquillo & $13^{\circ} 55^{\prime} 18,51^{\prime \prime}$ & " $76^{\circ} 21^{\prime} 02,59^{\prime \prime}$ & 3 & 2002 \\
\hline 57 & Ica & La Catedral & $13^{\circ} 56^{\prime} 10,33^{\prime \prime}$ & " 76º'17'09,44" & 2 & 2008 \\
\hline 58 & Ica & Supay & $13^{\circ} 57^{\prime} 30,42^{\prime \prime}$ & " 76º'16'20,18" & 2 & 2002 \\
\hline 59 & Ica & Pta. Sacasemita & $14^{\circ} 09^{\prime} 16,50^{\prime \prime}$ & " 76º'16'42,03" & 2 & 2009 \\
\hline 60 & Ica & Barlovento & $14^{\circ} 22^{\prime} 04,15^{\prime \prime}$ & " 76º6'05,57" & 1 & 2009 \\
\hline 61 & Ica & Pta. Olleros & $14^{\circ} 47^{\prime} 00,41^{\prime \prime}$ & " 7544'09,98" & 1 & 2009 \\
\hline 62 & Ica & Puerto Caballas & $14^{\circ} 56 ' 28,43^{\prime \prime}$ & " 75³0'00,17" & 2 & 2008 \\
\hline 63 & Ica & San Fernando (Fondeadero) & ) $15^{\circ} 08^{\prime} 10,31^{\prime \prime}$ & " 75²2'04,67" & 1 & 2005 \\
\hline 64 & Ica & San Fernando & $15^{\circ} 08^{\prime} 29,43^{\prime \prime}$ & " $75^{\circ} 22^{\prime} 05,57^{\prime \prime}$ & 1 & 2003 \\
\hline 65 & Ica & La Aguada & $15^{\circ} 08^{\prime} 48,71^{\prime \prime}$ & " 75¹9'44,96" & 1 & 2000 \\
\hline
\end{tabular}

\begin{tabular}{|c|c|c|c|c|c|c|}
\hline $\mathbf{N}^{\circ}$ & Dpto. & Localidad & Lat. S & Long. W & Reg. & Año \\
\hline 66 & Ica & Pta. Gallinazo & $15^{\circ} 09^{\prime} 01,64^{\prime \prime}$ & $75^{\circ} 20^{\prime} 48,90^{\prime \prime}$ & 1 & 2002 \\
\hline 67 & Ica & San Nicolás & $15^{\circ} 15^{\prime} 14,02^{\prime \prime}$ & $75^{\circ} 14 \cdot 56,51^{\prime \prime}$ & 1 & 2003 \\
\hline 68 & Ica & San Juanito & $15^{\circ} 16^{\prime} 21,37^{\prime \prime}$ & $75^{\circ} 14 ' 25,13^{\prime \prime}$ & 1 & 2003 \\
\hline 69 & Ica & Pta. San Juan & $15^{\circ} 21^{\prime} 50,88^{\prime \prime}$ & $75^{\circ} 11^{\prime} 30,06^{\prime \prime}$ & 2 & 2004 \\
\hline 70 & Arequipa & Tres Hermanas & $15^{\circ} 26^{\prime} 34,39^{\prime \prime}$ & $75^{\circ} 04^{\prime} 17,01^{\prime \prime}$ & 3 & 2002 \\
\hline 71 & Arequipa & Almeja & $15^{\circ} 26^{\prime} 34,75^{\prime \prime}$ & $75^{\circ} 04^{\prime} 05,21^{\prime \prime}$ & 2 & 2003 \\
\hline 72 & Arequipa & La Aguada & $15^{\circ} 27^{\prime} 31,43^{\prime \prime}$ & $75^{\circ} 02 ' 30,48^{\prime \prime}$ & 3 & 2002 \\
\hline 73 & Arequipa & Yanyarina & $15^{\circ} 27^{\prime} 33,60^{\prime \prime}$ & $75^{\circ} 01^{\prime} 21,15^{\prime \prime}$ & 2 & 2003 \\
\hline 74 & Arequipa & El Cable & $15^{\circ} 28^{\prime} 17,06^{\prime \prime}$ & $74^{\circ} 59^{\prime} 15,98^{\prime \prime}$ & 2 & 2003 \\
\hline 75 & Arequipa & Playa la Libertad & $15^{\circ} 28^{\prime} 47,64^{\prime \prime}$ & $74^{\circ} 58^{\prime} 58,08^{\prime \prime}$ & 5 & 2003 \\
\hline 76 & Arequipa & El Submarino & $15^{\circ} 29^{\prime} 06,41^{\prime \prime}$ & $74^{\circ} 58^{\prime} 40,68^{\prime \prime}$ & 1 & 2003 \\
\hline 77 & Arequipa & El Cachucho & $15^{\circ} 29^{\prime} 54,42^{\prime \prime}$ & $74^{\circ} 57^{\prime} 14,85^{\prime \prime}$ & 3 & 2003 \\
\hline 78 & Arequipa & Chala & $15^{\circ} 43^{\prime} 49,98^{\prime \prime}$ & $74^{\circ} 27^{\prime} 41,79^{\prime \prime}$ & 1 & 2008 \\
\hline 79 & Arequipa & Puerto Inca & $15^{\circ} 50^{\prime} 39,33^{\prime \prime}$ & $74^{\circ} 18^{\prime} 37,42^{\prime \prime}$ & 2 & 2008 \\
\hline 80 & Arequipa & $\begin{array}{l}\text { Pampa Redonda } \\
\text { (Fondeadero) }\end{array}$ & $16^{\circ} 00^{\prime} 00,05^{\prime \prime}$ & 74º1'35,73" & 2 & 2008 \\
\hline 81 & Arequipa & Pta. Pampa Redonda & $16^{\circ} 01^{\prime} 43,77^{\prime \prime}$ & $74^{\circ} 01^{\prime} 01,85^{\prime \prime}$ & 3 & 2008 \\
\hline 82 & Arequipa & Pta. Atico & $16^{\circ} 13^{\prime} 55,57^{\prime \prime}$ & $73^{\circ} 41^{\prime} 56,01^{\prime \prime}$ & 4 & 2003 \\
\hline 83 & Arequipa & Playa Oscuyo & $16^{\circ} 16^{\prime} 48,34^{\prime \prime}$ & $73^{\circ} 27^{\prime} 18,08^{\prime \prime}$ & 4 & 2003 \\
\hline 84 & Arequipa & La Planchada & $16^{\circ} 24^{\prime} 27,31^{\prime \prime}$ & $73^{\circ} 13^{\prime} 18,80^{\prime \prime}$ & 2 & 2009 \\
\hline 85 & Arequipa & Pta. Caleta & $16^{\circ} 30^{\prime} 54,79^{\prime \prime}$ & $72^{\circ} 57^{\prime} 42,83^{\prime \prime}$ & 2 & 2004 \\
\hline 86 & Arequipa & Punta El Uno & $16^{\circ} 30^{\prime} 56,75^{\prime \prime}$ & $72^{\circ} 566^{\prime} 51,86^{\prime \prime}$ & 1 & 2004 \\
\hline 87 & Arequipa & Pta. La Chira & $16^{\circ} 30^{\prime} 59,31^{\prime \prime}$ & $72^{\circ} 56^{\prime} 00,20^{\prime \prime}$ & 6 & 2004 \\
\hline 88 & Arequipa & Quilca & $16^{\circ} 42^{\prime} 53,40^{\prime \prime}$ & $72^{\circ} 26^{\prime} 06,48^{\prime \prime}$ & 1 & 2003 \\
\hline 89 & Arequipa & Pta. Arantas & $16^{\circ} 47^{\prime} 38,94^{\prime \prime}$ & 72º' $1955,03^{\prime \prime}$ & 1 & 2003 \\
\hline 90 & Arequipa & Pta. San José & $16^{\circ} 49^{\prime} 32,88^{\prime \prime}$ & $72^{\circ} 17 \cdot 56,43^{\prime \prime}$ & 3 & 2009 \\
\hline 91 & Arequipa & Pta. Hornillos & $16^{\circ} 52 ' 32,67^{\prime \prime}$ & $72^{\circ} 17^{\prime} 06,72^{\prime \prime}$ & 1 & 2004 \\
\hline 92 & Arequipa & Pto. Matarani & $16^{\circ} 59^{\prime} 43,14^{\prime \prime}$ & $72^{\circ} 06^{\prime} 19,10^{\prime \prime}$ & 4 & 2004 \\
\hline 93 & Arequipa & Pto. Viejo & $17^{\circ} 00^{\prime} 22,26^{\prime \prime}$ & $72^{\circ} 0634,97^{\prime \prime}$ & 3 & 2007 \\
\hline 94 & Arequipa & Caleta Islay & $17^{\circ} 00^{\prime} 57,66^{\prime \prime}$ & $72^{\circ} 06 ' 33,40^{\prime \prime}$ & 5 & 2003 \\
\hline 95 & Arequipa & Catarindo & $17^{\circ} 01^{\prime} 02,53^{\prime \prime}$ & $72^{\circ} 02^{\prime} 02,29^{\prime \prime}$ & 2 & 2003 \\
\hline 96 & Arequipa & Aguada Lima & $17^{\circ} 01^{\prime} 19,58^{\prime \prime}$ & $72^{\circ} 01^{\prime} 21,93^{\prime \prime}$ & 1 & 2003 \\
\hline 97 & Arequipa & Mollendo & $17^{\circ} 01^{\prime} 57,69^{\prime \prime}$ & $72^{\circ} 00^{\prime} 58,65^{\prime \prime}$ & 4 & 2003 \\
\hline 98 & Arequipa & Pta. Corio & $17^{\circ} 14^{\prime} 52,78^{\prime \prime}$ & $71^{\circ} 35^{\prime} 40,57^{\prime \prime}$ & 1 & 2002 \\
\hline 99 & Arequipa & Pta. Playuelas & $17^{\circ} 15^{\prime} 31,09^{\prime \prime}$ & $71^{\circ} 33^{\prime} 51,08^{\prime \prime}$ & 3 & 2003 \\
\hline 100 & Arequipa & Pta. Jesús y Cocotea & $17^{\circ} 16^{\prime} 02,17^{\prime \prime}$ & $71^{\circ} 31^{\prime} 38,23^{\prime \prime}$ & 1 & 2004 \\
\hline 101 & Moquegua & Playa Yerba Buena & $17^{\circ} 18^{\prime} 24,57^{\prime \prime}$ & $71^{\circ} 28^{\prime} 12,66^{\prime \prime}$ & 2 & 2009 \\
\hline 102 & Moquegua & Pta. Yerba Buena & $17^{\circ} 19^{\prime} 09,01^{\prime \prime}$ & $71^{\circ} 28127,77^{\prime \prime}$ & 3 & 2002 \\
\hline 103 & Moquegua & Pta. El Carmen & $17^{\circ} 21^{\prime} 44,58^{\prime \prime}$ & $71^{\circ} 25^{\prime} 46,12^{\prime \prime}$ & 1 & 2003 \\
\hline 104 & Moquegua & Pta. Callango & $17^{\circ} 23^{\prime} 04,38^{\prime \prime}$ & $71^{\circ} 24^{\prime} 18,20^{\prime \prime}$ & 4 & 2003 \\
\hline 105 & Moquegua & Platanales & $17^{\circ} 23^{\prime} 09,11^{\prime \prime}$ & $71^{\circ} 23 ' 53,87^{\prime \prime}$ & 1 & 2009 \\
\hline 106 & Moquegua & Pta Jaboncillo & $17^{\circ} 24^{\prime} 47,95^{\prime \prime}$ & $71^{\circ} 23^{\prime} 11,17^{\prime \prime}$ & 2 & 2010 \\
\hline 107 & Moquegua & Pocoma & $17^{\circ} 26^{\prime} 09,54^{\prime \prime}$ & $71^{\circ} 23^{\prime} 01,97^{\prime \prime}$ & 1 & 2010 \\
\hline 108 & Moquegua & Miraflores & $17^{\circ} 28 ' 30,18^{\prime \prime}$ & $71^{\circ} 22^{\prime} 20,64^{\prime \prime}$ & 2 & 2010 \\
\hline 109 & Moquegua & Chuza & $17^{\circ} 29^{\prime} 10,93^{\prime \prime}$ & $71^{\circ} 21^{\prime} 51,12^{\prime \prime}$ & 1 & 2004 \\
\hline 110 & Moquegua & Ilo & $17^{\circ} 38 ' 36,64^{\prime \prime}$ & $71^{\circ} 20^{\prime} 48,69^{\prime \prime}$ & 1 & 2003 \\
\hline 111 & Moquegua & Pto. Ingles & $17^{\circ} 39^{\prime} 47,78^{\prime \prime}$ & $71^{\circ} 21^{\prime} 29,28^{\prime \prime}$ & 1 & 2002 \\
\hline 112 & Moquegua & Corralitos & $17^{\circ} 40^{\prime} 15,35^{\prime \prime}$ & $71^{\circ} 21^{\prime} 45,21^{\prime \prime}$ & 2 & 2002 \\
\hline 113 & Moquegua & Pta. Coles & $17^{\circ} 42^{\prime} 10,71^{\prime \prime}$ & $71^{\circ} 22^{\prime} 46,42^{\prime \prime}$ & 5 & 2004 \\
\hline 114 & Moquegua & La Hondonada & $17^{\circ} 46^{\prime} 36,60^{\prime \prime}$ & $71^{\circ} 11^{\prime} 34,11^{\prime \prime}$ & 1 & 2008 \\
\hline 115 & Moquegua & El Alto & $17^{\circ} 46^{\prime} 45,92^{\prime \prime}$ & $71^{\circ} 11^{\prime} 18,54^{\prime \prime}$ & 1 & 2008 \\
\hline 116 & Moquegua & Playa La Higuera & $17^{\circ} 48^{\prime} 48,24^{\prime \prime}$ & $71^{\circ} 09^{\prime} 44,01^{\prime \prime}$ & 5 & 2008 \\
\hline 117 & Moquegua & Pta. Chorrillos & $17^{\circ} 49^{\prime} 03,53^{\prime \prime}$ & $71^{\circ} 09^{\prime} 15,50^{\prime \prime}$ & 2 & 2009 \\
\hline 118 & Moquegua & Pta. Icuy & $17^{\circ} 49^{\prime} 16,79^{\prime \prime}$ & $71^{\circ} 0831,86^{\prime \prime}$ & 3 & 2009 \\
\hline 119 & Tacna & Pta. Picata & $17^{\circ} 52^{\prime} 03,96^{\prime \prime}$ & $71^{\circ} 05^{\prime} 50,06^{\prime \prime}$ & 3 & 2002 \\
\hline 120 & Tacna & Santa Rosa & $17^{\circ} 52^{\prime} 46,13^{\prime \prime}$ & $71^{\circ} 03^{\prime} 16,23^{\prime \prime}$ & 2 & 2009 \\
\hline 121 & Tacna & La Meca & $17^{\circ} 57^{\prime} 03,74^{\prime \prime}$ & $70^{\circ} 54 ' 42,86^{\prime \prime}$ & 2 & 2002 \\
\hline 122 & Tacna & Pto. Grau & $17^{\circ} 59^{\prime} 35,98^{\prime \prime}$ & $70^{\circ} 53^{\prime} 03,94^{\prime \prime}$ & 8 & 2002 \\
\hline 123 & Tacna & C.A. FONDEPES & $17^{\circ} 59^{\prime} 59,79^{\prime \prime}$ & $70^{\circ} 53^{\prime} 10,65^{\prime \prime}$ & 3 & 2009 \\
\hline 124 & Tacna & Morro de Sama & $18^{\circ} 00^{\prime} 15,46^{\prime \prime}$ & $70^{\circ} 53^{\prime} 18,18^{\prime \prime}$ & 4 & 2003 \\
\hline 125 & Tacna & La Lobera & $18^{\circ} 00^{\prime} 37,57^{\prime \prime}$ & $70^{\circ} 52^{\prime} 54,68^{\prime \prime}$ & 2 & 2009 \\
\hline 126 & Tacna & Pta. Curimani & $18^{\circ} 01^{\prime} 13,00^{\prime \prime}$ & $70^{\circ} 51^{\prime} 48,00^{\prime \prime}$ & 1 & 2002 \\
\hline 127 & Tacna & Qda. de los Burros & $18^{\circ} 01^{\prime} 55,76^{\prime \prime}$ & $70^{\circ} 50^{\prime} 09,74^{\prime \prime}$ & 2 & 2009 \\
\hline 128 & Tacna & Pta. Balconcillo & $18^{\circ} 02^{\prime} 02,79^{\prime \prime}$ & $70^{\circ} 49^{\prime} 44,52^{\prime \prime}$ & 3 & 2009 \\
\hline 129 & Tacna & Canepa & $18^{\circ} 05^{\prime} 23,83^{\prime \prime}$ & $70^{\circ} 45^{\prime} 09,08^{\prime \prime}$ & 1 & 2009 \\
\hline 130 & Tacna & Pto. Vila Vila & $18^{\circ} 07^{\prime} 05,88^{\prime \prime}$ & $70^{\circ} 43^{\prime} 39,44^{\prime \prime}$ & 6 & 2002 \\
\hline
\end{tabular}

